\title{
DIFFERENTIAL GAMES \\ WITH LIPSCHITZ CONTROL FUNCTIONS AND APPLICATIONS TO GAMES WITH PARTIAL DIFFERENTIAL EQUATIONS( ${ }^{1}$ )
}

BY

\section{EMMANUEL NICHOLAS BARRON}

\begin{abstract}
In $\$ 1$ we formulate a differential game when the dynamics is the inhomogeneous heat equation. In $\$ 2$ we state the basic theory of differential games when the controls must choose uniformly Lipschitz control functions. We then prove some general theorems for the case when the controls may choose any measurable control functions. These theorems hold for games with any dynamics. In $\$ 3$ we apply our theory developed to our particular example and in $\$ 4$ we prove the existence of value for games with partial differential equations.
\end{abstract}

Introduction. For constants $M, L \geqslant 0$, let $V^{+}(M, L)$ denote the upper value of a differential game with any dynamics of fixed duration when the player $y$ is restricted to choosing control functions which are Lipschitz $(M)$ and the player $z$ is restricted to choosing control functions which are Lipschitz $(L)$. We will show that a differential game has value $V$ (in the sense of Friedman [1], [2]) if $\lim _{L \rightarrow \infty} \lim _{M \rightarrow \infty} V^{+}(M, L)=\lim _{M \rightarrow \infty} \lim _{L \rightarrow \infty} V^{+}(M, L)$; furthermore $V=$ $\lim _{M \rightarrow \infty} \lim _{L \rightarrow \infty} V^{+}(M, L)$.

For the particular case of games with ordinary differential equations we will show that the equality of the iterated limits is necessary as well as sufficient; furthermore, if the value, $V$, exists then the double $\operatorname{limit}_{(M, L) \rightarrow(\infty, \infty)} V^{+}(M, L)$ exists and equals $V$.

Finally, for games with ordinary differential equations, Friedman [2] , [3], Elliott-Kalton [5] and Fleming [6] have shown the existence of value when the so-called Isaacs minimax condition holds. Using the results of the present

Received by the editors May 29, 1974 and, in revised form, October 16, 1974. AMS (MOS) subject classifications (1970). Primary 90D25; Secondary 35K05, 39A10. Key words and phrases. Lipschitz differential game, differential games with partial differential equations, Isaacs' condition.

1 This paper consists of part of the author's doctoral dissertation at Northwestern University prepared under the direction of Professor Avner Friedman to whom I am grateful. I would also like to thank Robert Jensen for many helpful discussions. 
paper mentioned above, we shall extend the Friedman-Elliott-Kalton-Fleming theorem to games with partial differential equations.

1. Preliminaries. We denote the $m$-dimensional Euclidean space by $R^{m}$. Consider the initial-boundary value problem:

$$
\begin{aligned}
\partial u / \partial t=\partial^{2} u / \partial x^{2}+f(y, z) & \quad(0<t \leqslant T, 0<x<B), \\
u(0, x) & =\phi(x), \\
u(t, 0) & =a(t), \\
u(t, B) & =b(t) .
\end{aligned}
$$

Let $Y, Z$ be given compact subsets of some $R^{p}, R^{q}$ respectively. We assume

$$
f \text { is Lipschitz continuous in }(y, z) \in Y \times Z \text {, }
$$

$$
\phi \text { is continuous in } x \in[0, B] \text {, }
$$

$a$ and $b$ are continuous in $t \in[0, T]$.

$$
\phi(0)=a(0), \phi(B)=b(0) \text {. }
$$

The set $Y(Z)$ is called the control set for the "player" $y(z)$. A measurable function $y(t, x)(z(t, x))$ with values in $Y(Z)$ for almost all $(t, x) \in[0, T]$ $x[0, B]$ is called a control function for $y(z)$. If we substitute in (1.1) any control functions $y=y(t, x), z=z(t, x)(0<t \leqslant T, 0<x<B)$ we obtain a differential equation:

$$
\partial u / \partial t=\partial^{2} u / \partial x^{2}+f(y(t, x), z(t, x)) \quad(0<t \leqslant T, 0<x<B) .
$$

Denote by $G$ the Green's function for the problem (1.1)-(1.4). When $y$ and $z$ have chosen the control functions $y(t, x)$ and $z(t, x)$, respectively, then a solution of (1.2)-(1.5) is given by

$u(t, x)=\int_{0}^{T} \int_{0}^{B} G(t, x ; \tau, \xi) f(y(\tau, \xi), z(\tau, \xi)) d \xi d \tau$

$$
+\int_{0}^{B} G(t, x ; 0, \xi) \phi(\xi) d \xi+\int_{0}^{T}\left\{\left.\frac{\partial G}{\partial \xi}\right|_{\xi=0} a(\tau)-\left.\frac{\partial G}{\partial \xi}\right|_{\xi=B} b(\tau)\right\} d \tau .
$$

The condition (A) insures that each integral appearing in (1.6) is well defined. Note that $u$ given by $(1.6)$ is continuous on $[0, T] \times[0, B]$ and satisfies (1.5) almost everywhere. We call $u(t, x)$ the trajectory corresponding to $y(t, x), z(t, x)$.

We introduce a functional, called a payoff functional:

$$
P(y, z)=\int_{0}^{T} \int_{0}^{B} h(t, x, u(t, x), y(t, x), z(t, x)) d x d t
$$

where $h$ is a given function.

We shall assume: 


$$
h(t, x, u, y, z) \text { is uniformly Lipschitz continuous on }
$$

$$
[0, T] \times[0, B] \times R^{1} \times Y \times Z .
$$

Unless otherwise noted, conditions (A) and (B) will hold throughout this paper.

The condition (B) insures that the payoff (1.7) is well defined for each particular choice of control functions $y(t, x), z(t, x)$. The aim of $y$ will be to maximize the payoff and the aim of $z$ will be to minimize it. The precise circumstances under which these two conflicting aims are to be carried out when $y$ and $z$ choose measurable control functions is explained in Friedman [1], [2]. We shall use and conform to Friedman's notation. Thus, for example, we use his concepts of upper $\delta$-value, $V^{\delta}$, lower $\delta$-value $V_{\delta}$, upper value, $V^{+}=\lim _{\delta \rightarrow 0} V^{\delta}$ and lower value $V^{-}=\lim _{\delta \rightarrow 0} V_{\delta}$. Here, for $\alpha$ any positive integer, we set $\delta=$ $T / \alpha$ and partition the interval $[0, T]$ into $\alpha$ subintervals $I_{j}$ of length $\delta$ :

$$
\begin{aligned}
& I_{1}=\left\{t \in[0, T]: 0 \leqslant t \leqslant \delta_{1}\right\}, \\
& I_{j}=\left\{t \in[0, T]: \delta_{j-1}<t \leqslant \delta_{j}\right\} \quad(2 \leqslant j \leqslant \alpha),
\end{aligned}
$$

where $\delta_{j}=j \cdot \delta, 1 \leqslant j \leqslant \alpha, \delta_{0}=0, \delta_{\alpha}=T$. Also $Y_{j}$ and $Z_{j}$ are the classes of measurable functions defined on $I_{j} \times[0, B]$ whose values are almost everywhere, contained in $Y, Z$, respectively. Proceed as in Friedman. Finally, $\delta$-strategies $\Gamma^{\delta}, \Gamma_{\delta}$ for $y$ and $\Delta^{\delta}, \Delta_{\delta}$ for $z$ are as defined in Friedman [1], [2].

Following Friedman, we say the "game associated with (1.1)-(1.4) and payoff (1.7) has value, $V$, if $V^{+}=V^{-}$. Then $V^{+} \equiv V$."

We shall use all of the results quoted in Friedman [1], [2] relating to $V^{\delta}$, $V_{\delta}, V^{+}, V^{-}$.

2. Differential games with Lipschitz control functions. We shall state and prove the results of this section for the dynamics (1.1)-(1.4) and payoff (1.7) although the results are valid for games with ordinary differential and integral equations as dynamics. For other dynamics the proofs are similar. Let $\left.f\right|_{A}$ denote the function $f$ restricted to the set $A$.

Notation. Given positive constants $M, L$, let $Y^{M}(I \times J), Z^{L}(I \times J)$ be the classes of all functions defined on the cross product $I \times J$ of intervals $I$ and $J \subset R^{1}$ which are uniformly Lipschitz continuous on $I \times J$ with Lipschitz constant $M$ and $L$, respectively, and whose values are in given compact subsets $Y \subset$ $R^{p}$ and $Z \subset R^{q}$, respectively. A function in $Y^{m}(I \times J)\left(Z^{L}(I \times J)\right)$ will be denoted by $y_{M}=y_{M}(t, x) \quad\left(z_{L}=z_{L}(t, x)\right)$.

Let $Y_{0}^{M}(I \times J)\left(Z_{0}^{L}(I \times J)\right)$ denote the subclass of functions in $Y^{M}(I \times J)$ $\left(Z^{L}(I \times J)\right)$ which are some fixed element of $Y(Z)$, say $\bar{y}(\bar{z})$, at $(0, x)$ (if $(0, x) \in I \times J)$.

REMARK 2.1. 1. By the Arzéla-Ascoli lemma, $Y^{M}([0, T] \times[0, B])$ and 
$Z^{L}([0, T] \times[0, B])$ are compact in the topology of continuous functions (or in $L^{p}, 1 \leqslant p \leqslant \infty, L^{p}$ denotes the usual Lebesgue spaces). Since $Y_{0}^{M}([0, T] \times$ $[0, B])$ and $Z_{0}^{L}([0, T] \times[0, B])$ are closed sets, they are also compact.

2. In the above notational definitions we could require that $\bar{y}, \bar{z}$ be fixed functions of $x$, i.e. $\bar{y}=\bar{y}(0, x), \bar{z}=\bar{z}(0, x), x \in[0, B]$, but the added restriction above is more convenient. The proofs go through with $\bar{y}, \bar{z}$ fixed functions of $x$.

3. In this paper, for convenience we deal with the intervals $[0, T]$ and $[0, B]$, however we can certainly use other initial points $\left(t_{0}, x_{0}\right)$ so that we are on the rectangle $\left[t_{0}, T\right] \times\left[x_{0}, B\right]$. In this case $\bar{y}=\bar{y}\left(t_{0}, x\right), \bar{z}=\bar{z}\left(t_{0}, x\right)$.

When one or both players in a differential game must choose a Lipschitz function, then we call such a game a Lipschitz differential game. We state the setting in the following.

Partition $[0, T]$ into the $\alpha$ subintervals of equal length $\delta=T / \alpha$ given by (1.8), (1.9). Let $Y_{j}^{M}=Y^{M}\left(I_{j} \times[0, B]\right), Z_{j}^{L}=Z^{L}\left(I_{j} \times[0, B]\right)$.

Let ${ }^{M} \Gamma^{\delta, j}$ be any map from $Z_{1} \times Y_{1}^{M} \times Z_{2} \times Y_{2}^{M} \times \cdots \times Z_{j-1} \times$ $Y_{j-1}^{M} \times Z_{j}$ into $Y_{j}^{M}$ and let ${ }^{M} \Gamma^{\delta}=\left({ }^{M} \Gamma^{\delta, 1}, \ldots,{ }^{M} \Gamma^{\delta, \alpha}\right)$.

Let ${ }^{L} \Delta^{\delta, j}$ be any map from $Y_{1} \times Z_{1}^{L} \times Y_{2} \times Z_{2}^{L} \times \cdots \times Y_{j-1} \times Z_{j-1}^{L}$ $\times Y_{j}$ into $Z_{j}^{L}$ and let ${ }^{L} \Delta^{\delta}=\left({ }^{L} \Delta^{\delta, 1}, \ldots, L \Delta^{\delta, \alpha}\right)$.

Let ${ }^{M} \Gamma_{\delta, 1}$ denote any function in $Y_{1}^{M}$ and let ${ }^{M} \Gamma_{\delta, j}(2 \leqslant j \leqslant \alpha)$ be any map from $Y_{1}^{M} \times Z_{1} \times \cdots \times Y_{j-1}^{M} \times Z_{j-1}$ into $Y_{j}^{M}$. Let ${ }^{M} \Gamma_{\delta}=\left({ }^{M} \Gamma_{\delta, 1}, \ldots\right.$, $\left.{ }^{M} \Gamma_{\delta, \alpha}\right)$

Let ${ }^{L} \Delta_{\delta, 1}$ denote any function in $Z_{1}^{L}$ and let ${ }^{L} \Delta_{\delta, j}(2 \leqslant j \leqslant \alpha)$ be any map from $Z_{1}^{L} \times Y_{1} \times \cdots \times Z_{j-1}^{L} \times Y_{j-1}$ into $Z_{j}^{L}$. Let ${ }^{L} \Delta_{\delta}=\left({ }^{L} \Delta_{\delta, 1}, \ldots\right.$, $\left.{ }^{L} \Delta_{\delta, \alpha}\right)$.

Given any pair $\left({ }^{L} \Delta_{\delta},{ }^{M} \Gamma^{\delta}\right)$ we can uniquely construct control functions $y_{M}^{\delta}(t, x)$ and $z_{L, \delta}(t, x)$ with components $y_{M, j}, z_{L, j}$ on $I_{j} \times[0, B]$ by $z_{L, 1}=$ ${ }^{L} \Delta_{\delta, 1}, y_{M, 1}={ }^{M} \Gamma^{\delta, 1}\left(z_{L, 1}\right)$ and for $2 \leqslant j \leqslant \alpha$

$$
\begin{aligned}
& z_{L, j}={ }^{L} \Delta_{\delta, j}\left(z_{L, 1}, y_{M, 1}, \ldots, z_{L, j-1}, y_{M, j-1}\right), \\
& y_{M, j}={ }^{M} \Gamma^{\delta, j}\left(z_{L, 1}, y_{M, 1}, \ldots, z_{L, j-1}, y_{M, j-1}, z_{L, j}\right) .
\end{aligned}
$$

We call a vector ${ }^{M} \Gamma^{\delta}\left({ }^{L} \Delta_{\delta}\right)$ such that the function $y_{M}^{\delta}(t, x)\left(z_{L, \delta}(t, x)\right)$ is a member of $Y^{M}([0, T] \times[0, B])\left(Z^{L}([0, T] \times[0, B])\right)$ an upper $\delta-(M)$ strategy for $y$ (lower $\delta-(L)$ strategy for $z$ ).

We call the pair $\left(y_{M}^{\delta}, z_{L, \delta}\right)$ the $(M, L)$-outcome, or simply the outcome of the pair of $\delta$-strategies $\left({ }^{L} \Delta_{\delta},{ }^{M} \Gamma^{\delta}\right)$. We write the corresponding payoff (1.7) in the form 


$$
\begin{aligned}
P\left(y_{M}^{\delta}, z_{L, \delta}\right) & \equiv P\left[{ }^{L} \Delta_{\delta},{ }^{M} \Gamma^{\delta}\right] \\
& \equiv P\left[{ }^{L} \Delta_{\delta, 1},{ }^{M} \Gamma^{\delta, 1}, \ldots,{ }^{L} \Delta_{\delta, \alpha},{ }^{M} \Gamma^{\delta, \alpha}\right] .
\end{aligned}
$$

We call the above scheme an upper $\delta-(M, L)$ game and denote it by ${ }^{M, L} G^{\delta}$. Thus, in $M, L G^{\delta}, y$ chooses an upper $\delta$ - $(M)$ strategy ${ }^{M} \Gamma^{\delta}, z$ chooses a lower $\delta$ - (L) strategy ${ }^{L} \Delta_{\delta}$ and they "play" according to (2.1), (2.2). The payoff of this "play" is given by (2.3). The number

$$
\begin{aligned}
& V^{\delta}(M, L)=\inf _{L_{\Delta_{\delta, 1}}} \sup _{\Gamma^{\delta, 1}} \cdots \inf _{L_{\Delta_{\delta, \alpha}} M_{\Gamma^{\delta, \alpha}}} \sup P{ }^{L} \Delta_{\delta, 1}, M^{M} \Gamma^{\delta, 1}, \\
& \left.\ldots, L_{\Delta_{\delta, \alpha}},{ }^{M} \Gamma^{\delta, \alpha}\right]
\end{aligned}
$$

is called the upper $\delta \cdot(M, L)$ value of the game ${ }^{M, L} G^{\delta}$.

Similarly we define the concept of a lower $\delta-(M, L)$ game ${ }^{M, L} G_{\delta}$ and lower $\delta-(M, L)$ value $V_{\delta}(M, L)$. Here $y$ chooses a lower $\delta-(M)$ strategy ${ }^{M} \Gamma_{\delta}, z$ chooses an upper $\delta-(L)$ strategy ${ }^{L} \Delta^{\delta}$ and they play in a fashion similar to (2.1), (2.2).

Then $V_{\delta}(M, L)$ is given by

$$
V_{\delta}(M, L)=\sup _{M_{\Gamma_{\delta, 1}}} \inf _{L_{\Delta^{\delta, 1}}} \cdots \sup _{{ }_{\Gamma_{\delta, \alpha}}} \inf _{L_{\Delta^{\delta, \alpha}}} P\left[{ }^{M} \Gamma_{\delta, 1},{ }^{L} \Delta^{\delta, 1}, \ldots,{ }^{M} \Gamma_{\delta, \alpha},{ }^{L} \Delta^{\delta, \alpha}\right]
$$

Note that our definitions of lower $\delta$-(M) strategies, ${ }^{M} \Gamma_{\delta}$, and upper $\delta$-(L) strategies, ${ }^{L} \Delta^{\delta}$, require that the outcome functions $y_{M, \delta}=y_{M, \delta}(t, x)$ and $z_{L}^{\delta}=$ $z_{L}^{\delta}(t, x)$ be members of $Y^{M}([0, T] \times[0, B])$ and $Z^{L}([0, T] \times[0, B])$, respectively.

Definition. Denote by $V^{\delta}(M) \equiv V^{\delta}(M, \infty)\left(V_{\delta}(M) \equiv V_{\delta}(M, \infty)\right)$ the upper $\delta$ - $(M, \infty)$ value (lower $\delta-(M, \infty)$ value) of the $(M, \infty)$-differential game. That is, in the $(M, \infty)$-game, $z$ can choose any measurable control function.

Similarly, denote by $V^{\delta}(L) \equiv V^{\delta}(\infty, L) \quad\left(V_{\delta}(L) \equiv V_{\delta}(\infty, L)\right)$ the upper $\delta-(\infty, L)$ value (lower $\delta \cdot(\infty, L)$ value) of the $(\infty, L)$-differential game. Thus, in the $(\infty, L)$-game, $y$ chooses any measurable control function.

Definition. The pair of sequences ${ }^{M, L} G \equiv\left(\left\{{ }^{M, L} G^{\delta}\right\},\left\{{ }^{M, L} G_{\delta}\right\}\right), \delta=$ $T / \alpha, \alpha=1,2, \ldots$, is called the $(M, L)$-differential game associated with the initial-boundary value problem (1.1)-(1.4) and payoff (1.7).

If $\lim _{\delta \rightarrow 0} V^{\delta}(M, L) \quad\left(\lim _{\delta \rightarrow 0} V_{\delta}(M, L)\right)$ exists, we call it the upper $(M, L)$-value (lower $(M, L)$-value) of the $(M, L)$-differential game ${ }^{M, L} G$ and denote it by $V^{+}(M, L) \equiv \lim _{\delta \rightarrow 0} V^{\delta}(M, L)\left(V^{-}(M, L) \equiv \lim _{\delta \rightarrow 0} V_{\delta}(M, L)\right)$.

If $V^{ \pm}(M, L)$ exist and $V^{+}(M, L)=V^{-}(M, L)$, then we denote $V^{+}(M, L)$ by $V(M, L)$ and call it the $(M, L)$-value of the $(M, L)$-differential game.

REMARK 2.2. The definitions and results for $V^{\delta}(M, L), V_{\delta}(M, L)$ are similar to Friedman's definitions and results for $V^{\delta}, V_{\delta}$ in [1], [2]. The proof exten- 
sions to $V^{\delta}(M, L), V_{\delta}(M, L)$ are clear and we shall consider them established and shall use the results throughout. In particular $V^{ \pm}(M, L)$ exists uniformly in $(M, L)$.

LEMMA 2.1. For each $M \geqslant 0, L \geqslant 0$,

(i) $V^{+}(L) \geqslant V^{+}(M, L) \geqslant V^{-}(M, L) \geqslant V^{-}(M)$,

(ii) $V^{+}(L) \geqslant V^{+}(M, L) \geqslant V^{+}(M)$,

(iii) $M \geqslant M^{\prime} \geqslant 0$ implies $V^{ \pm}(M, L) \geqslant V^{ \pm}\left(M^{\prime}, L\right)$,

(iv) $L \geqslant L^{\prime} \geqslant 0$ implies $V^{ \pm}(M, L) \leqslant V^{ \pm}\left(M, L^{\prime}\right)$.

The proof of Lemma 2.1 is immediate upon comparing classes of control functions.

The main purpose of this section is to establish the following:

THEOREM 2.1. Let (A), (B) hold. If

$$
\lim _{M \rightarrow \infty} \lim _{L \rightarrow \infty} V^{+}(M, L)=\lim _{L \rightarrow \infty} \lim _{M \rightarrow \infty} V^{+}(M, L)
$$

then the differential game associated with (1.1)-(1.4) and payoff (1.7) has value V, i.e. $V=V^{+}=V^{-}$and

$$
V=\lim _{L \rightarrow \infty} \lim _{M \rightarrow \infty} V^{+}(M, L)=\lim _{M \rightarrow \infty} \lim _{L \rightarrow \infty} V^{+}(M, L) .
$$

Note that by Lemma 2.1 each iterated limit in (2.5) exists.

For simplicity we will prove Theorem 2.1 in the case $B=T$ although the proof can be carried out in general.

We introduce various intermediate concepts of values in which the controls will be restricted to choosing various types of functions. We also will introduce a double partition on the interval $[0, T]$ which we shall use in introducing still more types of values. In the following definitions of these various types of values we give only a formal description. The precise details are tedious and are similar to those given above and in Friedman [1], [2].

Partition $[0, T] \times[0, B]$ into $\delta$-squares, $\delta=T / \alpha, \alpha$ a positive integer:

$$
\begin{gathered}
I_{1} \times I_{k}=\left[0, \delta_{1}\right] \times I_{k}, \\
I_{j} \times I_{k}=\left\{(t, x) \in[0, T] \times[0, B]: \delta_{j-1}<t \leqslant \delta_{j}, \delta_{k-1}<x \leqslant \delta_{k}\right\}
\end{gathered}
$$

for $1 \leqslant j, k \leqslant \alpha, \delta_{j}=j \cdot \delta, \delta_{0}=0, \delta_{\alpha}=T=B$. We call $I_{j} \times I_{k}$ a $\delta$-square.

Partition $[0, T]$ also into $\eta$-intervals, $\eta=T / \beta, \beta$ a positive integer: $K_{1}=$ $\left[0, \eta_{1}\right], K_{r}=\left\{t \in[0, T]: \eta_{r-1}<t \leqslant \eta_{r}\right\}$ for $2 \leqslant r \leqslant \beta, \eta_{r}=r \cdot \eta, \eta_{0}=0$, $\eta_{\beta}=T$.

All the control functions below take values in the given compact sets $Y$ or $Z$. If the controls are measurable we mean this in the sense of almost everywhere. If the controls are continuous we mean everywhere.

Definition. (i) ${ }_{y} V^{\delta}$-upper $\delta$-value with $y$ choosing control functions 
which are constants on $\delta$-squares. That is, on each $I_{j} \times I_{k}, y=y(t, x)$ will be a constant. The corresponding $\delta$-strategies are denoted by $\Delta_{\delta},{ }^{y} \Gamma^{\delta}$. Note that for a pair $\left(\Delta_{\delta},{ }^{y} \Gamma^{\delta}\right)$ the $z$-outcome is a measurable function.

Similarly ${ }_{z} V^{\delta}$ denotes the upper $\delta$-value with $z$ choosing controls constant on $\delta$-squares. The corresponding $\delta$-strategies are denoted by ${ }^{2} \Delta_{\delta}, \Gamma^{\delta}$. Here the $y$-outcome of a pair $\left({ }^{2} \Delta_{\delta}, \Gamma^{\delta}\right)$ is a measurable function.

(ii) $y, \delta V^{\eta}$-upper $\eta$-value with $y$ choosing control functions which are constants on $\delta$-squares. The $\eta$-strategies are denoted by $\Delta_{\eta}, y, \delta . \Gamma^{\eta}$. The $z$-outcome of a pair is measurable.

Similarly for ${ }_{z, \delta} V^{\eta}, z$ chooses constants on $\delta$-squares. The corresponding $\eta$-strategies are denoted by ${ }^{z, \delta} \Delta_{\eta}, \Gamma^{\eta}$.

(iii) ${ }_{y} V^{\delta}(L)$-upper $\delta$-value with $y$ choosing constants on $\delta$-squares and $z$ choosing elements of $Z_{0}^{L}([0, T] \times[0, B])$. Denote the $\delta$ strategies by ${ }^{L}{ }_{\Delta_{\delta}},{ }^{y} \Gamma^{\delta}$. Similarly for ${ }_{z} V^{\delta}(M), z$ chooses constants on $\delta$-squares and $y$ chooses from $Y_{0}^{M}([0, T] \times[0, B])$. The $\delta$-strategies are denoted by ${ }^{2} \Delta_{\delta},{ }^{M} \Gamma^{\delta}$.

(iv) $y, \delta V^{\eta}(L)$-upper $\eta$-value with $y$ and $z$ choosing as in (iii). Denote the $\eta$-strategies by ${ }^{L}{ }_{\Delta_{\eta}}^{0},{ }^{y, \delta} \Gamma^{\eta}$.

Similarly for ${ }_{z, \delta} V^{\eta}(M)$. Denote the corresponding strategies by ${ }^{z, \delta} \Delta_{\eta}$,
$M_{\Gamma^{\eta}}^{0}$.

Similarly we define lower $\delta$ - and $\eta$-values and strategies.

We offer some explanation for $y, \delta V^{\eta}$. The others are similar. In this upper $\eta$-game, $z$ moves first on $\left[0, \eta_{1}\right]$ and chooses a measurable function defined on $\left[0, \eta_{1}\right] \times[0, B]$. Then $y$ moves on $\left[0, \eta_{1}\right]$ by choosing a function, say $y_{1}$, defined on $\left[0, \eta_{1}\right] \times[0, B]$ and which is a constant on each $\left[0, \eta_{1}\right] \times I_{j}, 1 \leqslant j$ $\leqslant \alpha$. Thus $y_{1}=y_{1}(t, x)$ is a piecewise constant function on $\left[0, \eta_{1}\right] \times[0, B]$. This choice for $y$ in $\left[0, \eta_{1}\right]$ determines $y$ 's move in $\left(\eta_{1}, \eta_{2}\right],\left(\eta_{2}, \eta_{3}\right]$, etc. up to $\left(\eta_{l-1}, \eta_{l}\right]$ for some $1 \leqslant l \leqslant \alpha$, where $\left(\eta_{l-1}, \eta_{l}\right]$ is the first $\eta$-interval to contain the point $\delta_{1}, \eta_{l-1}<\delta_{1}<\eta_{l}$, since $y$ must choose the same function up to $\delta_{1}$. In $\left(\eta_{l-1}, \eta_{l}\right) y$ must choose the same function in $\left(\eta_{l-1}, \delta_{1}\right)$ but then may choose another piecewise constant function defined on $\left(\delta_{1}, \eta_{l}\right]$. Then $y$ must remain with this choice up to $\delta_{2}$ and the game proceeds.

We come to the basic lemma of this paper.

LEMMa 2.2. For every $\delta>0$
(i) $\lim _{\eta \rightarrow 0} z, \delta V^{\eta}={ }_{2} V^{\delta}$,
(ii) $\lim _{\eta \rightarrow 0} z, \delta V_{\eta}={ }_{2} V^{\delta}$, 

(iii) $\lim _{\eta \rightarrow 0}{ }_{y, \delta} V^{\eta}={ }_{y} V_{\delta}$,
(iv) $\lim _{\eta \rightarrow 0} y, \delta V_{\eta}={ }_{y} V_{\delta}$,

(v) $\lim _{\eta \rightarrow 0}{ }_{z, \delta} \stackrel{0}{V}^{\eta}(M)={ }_{2} \stackrel{0}{\delta}^{\delta}(M)$ uniformly in $M$,

(vi) $\lim _{\eta \rightarrow 0} \underset{z, \delta}{\stackrel{0}{V}}(M)={ }_{z} \stackrel{0}{V}^{\delta}(M)$ uniformly in $M$,

(vii) $\lim _{\eta \rightarrow 0} \stackrel{0}{{ }_{y, \delta}} \stackrel{V}{\eta}^{(L)}={ }_{y} \stackrel{0}{\delta}_{\delta}(L)$ uniformly in $L$,

(viii) $\lim _{\eta \rightarrow 0}{ }_{y, \delta} V_{\eta}(L)={ }_{y} V_{\delta}(L)$ uniformly in $L$,

Proof. (iii) We must show the following:

Fix $\delta>0$. Let $\epsilon>0$ be given. Then there is an $\eta_{0}(\epsilon)>0$ and

(2.6) there exists $\tilde{\Delta}_{\eta}$ such that $\eta \leqslant \eta_{0}$ implies ${ }_{y} V_{\delta} \geqslant P\left[\tilde{\Delta}_{\eta}(y), y\right]-\epsilon$ for all controls $y$ which are constants on $\delta$-squares;

(2.7) there exists ${ }^{y, \delta} \widetilde{\Gamma}^{\eta}$ such that $\eta \leqslant \eta_{0}$ implies ${ }_{y} V_{\delta} \leqslant P\left[z,{ }^{y, \delta} \widetilde{\Gamma}^{\eta}(z)\right]+$ $\epsilon$ for all controls $z$ which are measurable functions, with the $y$-outcome of $\left(z, y, \delta \widetilde{\Gamma}^{\eta}(z)\right)$ a function which is constant on $\delta$-squares.

Establishing (2.6), (2.7) yields (iii) in view of the fact that, as is easily seen,

$$
\begin{aligned}
y, \delta & V^{\eta}=\inf _{\Delta_{\eta}} \sup _{y} P\left[\Delta_{\eta}(y), y\right]=\sup _{y, \delta} \inf _{z} P\left[z,{ }^{y, \delta} \Gamma^{\eta}(z)\right], \\
{ }_{y} V_{\delta}= & \inf \sup _{\Delta^{\delta}} P\left[y, \Delta^{\delta}(y)\right]=\sup _{y_{\Gamma_{\delta}}} \inf _{z} P\left[\Gamma_{\delta}(z), z\right],
\end{aligned}
$$

where $y$ is constant on $\delta$-squares.

To show (2.6) we have that there exists an upper $\delta$-strategy $\widetilde{\Delta}^{\delta}$ for $z$ such that

$$
{ }_{y} V_{\delta} \geqslant P\left[y, \tilde{\Delta}^{\delta}(y)\right]-\epsilon
$$

for all controls $y$ constant on $\delta$-squares.

Let $\left(y, \tilde{z}^{\delta}\right)$ denote the outcome of $\left(y, \tilde{\Delta}^{\delta}(y)\right)$ given a control $y$ constant on $\delta$-squares. Note that $\tilde{z}^{\delta}$ is a measurable function.

Define the lower $\eta$-strategy $\widetilde{\Delta}_{\eta}$ for $z$ as follows:

Given any control $y$, constant on $\delta$-squares, let $y$ have components $y=$ $\left(y_{1}, \ldots, y_{\beta}\right)$. Let

$$
\widetilde{z}_{1}=\widetilde{\Delta}_{\eta, 1}=\bar{z}
$$

where $\bar{z}$ is an arbitrary point in the control set $Z$,

$$
\tilde{z}_{2}=\tilde{\Delta}_{\eta, 2}\left(\tilde{z}_{1}, y_{1}\right)=\left.\tilde{z}^{\delta}\right|_{K_{1} \times[0, B]}
$$


shifted to the right in $t$ by a distance $\eta$, and, for $3 \leqslant j \leqslant \beta$

$$
\tilde{z}_{j}=\tilde{\Delta}_{\eta, j}\left(\tilde{z}_{1}, y_{1}, \tilde{z}_{2}, y_{2}, \ldots, \tilde{z}_{j-1}, y_{j-1}\right)=\left.\tilde{z}^{\delta}\right|_{K_{j-1} \times[0, B]}
$$

shifted to the right in $t$ by a distance $\eta$. Here, as above, $K_{j}=\left\{t \in[0, T]: \eta_{j-1}\right.$ $\left.<t \leqslant \eta_{j}\right\}, \eta_{j}=j \cdot \eta, \eta=T / \beta$.

Let $\tilde{\Delta}_{\eta}=\left(\tilde{\Delta}_{\eta, 1}, \ldots, \tilde{\Delta}_{\eta, \beta}\right)$.

Let $\left(y, \tilde{z}_{\eta}\right)$ denote the outcome of $\left(y, \tilde{\Delta}_{\eta}(y)\right)$. Note that $\tilde{z}_{\eta}=\widetilde{z}_{\eta}(t, x)$ differs from $\tilde{z}^{\delta}=\widetilde{z}^{\delta}(t, x)$ only in that $\tilde{z}_{\eta}$ is a shift of $\tilde{z}^{\delta}$ by one interval $\left[0, \eta_{1}\right]$ of length $\eta$ to the right, and $\tilde{z}_{\eta}$ is arbitrary in $\left[0, \eta_{1}\right]: \tilde{z}_{\eta}(t-\eta, x)=\tilde{z}^{\delta}(t, x)$ for $(t, x) \in\left[\eta_{1}, T\right] \times[0, B]$. Thus, we can choose $\eta_{0}(\epsilon)$ such that $\eta_{0}(\epsilon)<\delta$ and

$$
\left.\left\|\tilde{z}_{\eta}-\tilde{z}^{\delta}\right\|_{L^{1}}<\epsilon \text { for } \eta \leqslant \eta_{0}(\epsilon)\right)
$$

Then, by elementary manipulations of (1.6), there is a positive function $\sigma(r)$ with $\sigma(r) \rightarrow 0$ as $r \rightarrow 0$ such that

$$
\left\|\tilde{u_{\delta}}-\tilde{u}^{\eta}\right\| \leqslant \sigma(\eta)<\epsilon \quad\left(\eta \leqslant \eta_{0}(\epsilon)\right),
$$

where $\tilde{u}_{\delta}, \tilde{u}^{\eta}$ are the trajectories corresponding to $\left(y, \tilde{z}^{\delta}\right)$ and $\left(y, \tilde{z}_{\eta}\right)$, respectively.

By (2.9), (2.10) and the continuity of $h$, there is another positive function $\sigma(r), \sigma(r) \rightarrow 0$ as $r \rightarrow 0$ such that for all controls $y$ constant on $\delta$-squares,

$$
\left|P\left(y, \tilde{z}^{\delta}\right)-P\left(y, \tilde{z}_{\eta}\right)\right| \leqslant \sigma(\eta)<\epsilon \text { when } \eta \leqslant \eta_{0}(\epsilon) .
$$

Combining this with (2.8) yields (2.6).

To show (2.7), we have that there exists a lower $\delta$-strategy for $y,{ }^{y} \widetilde{\Gamma}_{\delta}$ such that

$$
{ }_{y} V_{\delta} \leqslant P\left[{ }^{y} \widetilde{\Gamma}_{\delta}(z), z\right]+\epsilon
$$

for all measurable control functions $z$.

Given any control $z$, let $\left(\tilde{y}_{\delta}, z\right)$ denote the outcome of $\left({ }^{y} \widetilde{\Gamma}_{\delta}(z), z\right)$. Then $\tilde{y}_{\delta}$ is a function which is constant on $\delta$-squares, and $z$ is a measurable function.

Since every lower $\delta$-strategy can be considered to be an upper $\delta$-strategy, if we define for all $\eta,{ }^{y, \delta} \widetilde{\Gamma}^{\eta}={ }^{y} \widetilde{\Gamma}_{\delta}$ then ${ }^{y, \delta} \widetilde{\Gamma}^{\eta}$ is well defined as an upper $\eta$-strategy. Furthermore, the $y$-outcome of $\left(z,{ }^{y, \delta} \tilde{\Gamma}^{\eta}(z)\right)$ for any given control $z$ will be constant on $\delta$-squares. Thus by (2.11), for every $\eta$

$$
{ }_{y} V_{\delta} \leqslant P\left[z, y, \delta \widetilde{\Gamma}^{\eta}(z)\right]+\epsilon
$$

for all measurable control functions $z$, which is (2.7).

Proof of (iv). Since it is easily seen that ${ }_{y, \delta} V_{\eta} \geqslant{ }_{y} V_{\delta}$ for all $\eta$ smaller than $\delta$ we need only show that given $\epsilon>0$, there is $\eta_{0}(\epsilon)>0$ such that 


$$
0 \leqslant{ }_{y, \delta} V_{\eta}-{ }_{y} V_{\delta}<\epsilon \text { when } \eta \leqslant \eta_{0}(\epsilon)
$$

Note that

$$
{ }_{y, \delta} V_{\eta}=\sup _{y, \delta} \inf _{z} P\left[{ }^{y, \delta} \Gamma_{\eta}(z), z\right]
$$

Thus, there is a lower $\eta$-strategy ${ }^{y, \delta} \tilde{\Gamma}_{\eta}$ for $y$ such that

$$
y, \delta V_{\eta} \leqslant P\left[{ }^{y, \delta} \tilde{\Gamma}_{\eta}(z), z\right]+\epsilon
$$

for all measurable control functions $z$.

Given a measurable control $z$ with components $z=\left(z_{1}, \ldots, z_{\alpha}\right)$ we define the following lower $\delta$-strategy ${ }^{y} \widetilde{\Gamma}_{\delta}$ for $y$ :

The control $z=\left(z_{1}, \ldots, z_{\alpha}\right)$ has components $z=\left(\bar{z}_{1}, \ldots, \bar{z}_{\beta}\right)$ in the $\eta$-game $(\eta \leqslant \delta)$ which can be seen to be merely the restriction of the $\alpha$-components to $\eta$-intervals. Let $\left(z, \tilde{y}_{\eta}\right)$ denote the outcome of $\left(y, \delta \widetilde{\Gamma}_{\eta}(z), z\right)$. Then $\tilde{y}_{\eta}$ is constant on $\delta$-squares; say $\tilde{y}_{\eta}$ has components $\left(\tilde{y}_{1}, \ldots, \tilde{y}_{\alpha}\right)$ with each $\tilde{y}_{j}$ a piecewise constant function defined on $\left\{t: \delta_{j-1}<t \leqslant \delta_{j}\right\} \times[0, B]$. Let

$$
\begin{gathered}
y \tilde{\Gamma}_{\delta, 1}=\tilde{y}_{1},{ }^{y} \tilde{\Gamma}_{\delta, 2}\left(\tilde{y}_{1}, z_{1}\right)=\tilde{y}_{2}, \\
{ }^{y} \tilde{\Gamma}_{\delta, j}\left(\tilde{y}_{1}, z_{1}, \ldots, \tilde{y}_{j-1}, z_{j-1}\right)=\tilde{y}_{j} \text { for } 3 \leqslant j \leqslant \alpha .
\end{gathered}
$$

That is, given a control $z=\left(z_{1}, \ldots, z_{\alpha}\right)$, play $z$ against ${ }^{y, \delta} \widetilde{\Gamma}_{\eta}$. The $y$-outcome of this play is a function constant on $\delta$-squares. Define ${ }^{y} \widetilde{\Gamma}_{\delta}$ to be the strategy giving the same $y$-outcome.

Thus, by (2.13), with $\eta \leqslant \delta$,

$$
y, \delta V_{\eta} \leqslant P\left[{ }^{y} \Gamma_{\delta}(z), z\right]+\epsilon
$$

for all measurable controls $z$; which yields (2.12) in view of the fact that

$$
{ }_{y} V_{\delta}=\sup _{y_{\Gamma_{\delta}}} \inf P\left[{ }^{y} \Gamma_{\delta}(z), z\right]
$$

The proofs of (i), (ii), (v), (vi), (vii), (viii) are similar. Thus, in (vii) for example,

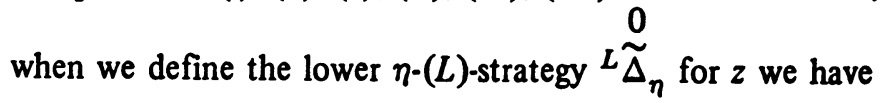

$$
\tilde{z}_{L, 1}=L \stackrel{0}{\tilde{\Delta}_{\eta, 1}}=\bar{z}
$$

where $\bar{z}$ is the point in $Z$ at which all the functions in $Z_{0}^{L}([0, T] \times[0, B])$ are determined at $(0, x)$. That is, for all $z_{L} \in Z_{0}^{L}([0, T] \times[0, B]) z_{L}(0, x)=\bar{z}$. Thus $\tilde{z}_{L, 1}(t, x)=\bar{z}$ for all $(t, x) \in\left[0, \eta_{1}\right] \times[0, B]$. Proceed to define ${ }^{L} \tilde{\Delta}_{\eta, j}$ 
as before by shifting the control function obtained from ${ }^{L} \stackrel{0}{\tilde{\Delta}^{\delta}}$ one interval in the $t$-axis of length $\eta$ to the right. Then, if $\tilde{z}_{L, \eta}$ denotes the $z$-outcome of $\left({ }^{L} \tilde{\Delta}_{\eta}(y), y\right)$ for a control $y$, constant on $\delta$-squares, where ${ }^{L} \tilde{\Delta}_{\eta}=\left({ }^{L} \tilde{\Delta}_{\eta, 1}^{0}, \ldots\right.$, 0 $\left.L \tilde{\Delta}_{\eta, \beta}\right)$, it is clear that

$$
\tilde{z}_{L, \eta} \in Z_{0}^{L}([0, T] \times[0, B])
$$

Again, we may choose $\eta_{0}(\epsilon)$ such that $\left\|\tilde{z}_{L, \eta}-\tilde{z}_{L}^{\delta}\right\|<\sigma(\eta)<\epsilon, \eta \leqslant \eta_{0}(\epsilon)$, for some positive function $\sigma(r), \sigma(r) \rightarrow 0$ as $r \rightarrow 0$, where $\widetilde{z}_{L}^{\delta}$ is the $z$-outcome of 0 $\left(y,{ }^{L} \tilde{\Delta}^{\delta}(y)\right)$. Proceed as in the proof of (iii).

Definition. Let ${ }_{y 0} V^{\delta}(M, L)$ denote the upper $\delta-(M, L)$ value of an $(M, L)$-differential game when $y$ must choose controls from $Y_{0}^{M}([0, T] \times[0, B])$ and $z$ chooses controls from $Z^{L}([0, T] \times[0, B])$. Similarly ${ }_{z 0} V^{\delta}(M, L)$ denotes the upper $\delta-(M, L)$ value when $z$ chooses controls from $Z_{0}^{L}([0, T] \times[0, B])$ and $y$ chooses controls from $Y^{M}([0, T] \times[0, B])$.

Let $\left.\stackrel{0}{V}^{\delta}(M) \equiv{ }_{{ }_{0}} V^{\delta}(M, \infty) \stackrel{0}{\left(V^{\delta}\right.}(L) \equiv{ }_{z 0} V^{\delta}(\infty, L)\right)$. Recall that in ${ }_{y 0} V^{\delta}(M, \infty)$, for example, $z$ can choose any measurable control function and $y$ chooses from $Y_{0}^{M}([0, T] \times[0, B])$.

Similarly we define lower $\delta$-values ${ }_{y 0} V_{\delta}(M, L),{ }_{z 0} V_{\delta}(M, L), \stackrel{0}{V_{\delta}}(M), \stackrel{0}{V_{\delta}}(L)$.

Again we remark that ${ }_{y_{0}} V^{ \pm}(M, L),{ }_{z 0} V^{ \pm}(M, L)$, etc. exist and we refer to Friedman [1], [2] as above.

LEMMA 2.3. For each $\delta>0$ and $\epsilon>0$

(i) There exists $L_{1}(\delta, \epsilon)$ such that

$$
{ }_{2, \delta} V^{\eta}+\epsilon / \delta^{2} \geqslant V^{\eta}(L) \text { for } L \geqslant L_{1}(\delta, \epsilon), \eta>0 .
$$

(ii) There exists $L_{2}(\delta, \epsilon)$ such that

$$
{ }_{2, \delta} V_{\eta}+\epsilon / \delta^{2} \geqslant V_{\eta}(L) \text { for } L \geqslant L_{2}(\delta, \epsilon), \eta>0 \text {. }
$$

(iii) There exists $M_{1}(\delta, \epsilon)$ such that

$$
V^{\eta}(M) \geqslant_{y, \delta} V^{\eta}-\epsilon / \delta^{2} \quad \text { for } M \geqslant M_{1}(\delta, \epsilon), \eta>0 \text {. }
$$

(iv) There exists $M_{2}(\delta, \epsilon)$ such that

$$
V_{\eta}(M) \geqslant_{y, \delta} V_{\eta}-\epsilon / \delta^{2} \text { for } M \geqslant M_{2}(\delta, \epsilon), \eta>0 .
$$


(v) There exists $L_{1}^{1}(\delta, \epsilon)$ such that ${ }_{z, \delta} \stackrel{0}{V}^{\eta}(M)+\epsilon / \delta^{2} \geqslant{ }_{y 0} V^{\eta}(M, L)$ for $L \geqslant L_{1}^{1}(\delta, \epsilon), M \geqslant 0, \eta>0$.

(vi) There exists $L_{2}^{1}(\delta, \epsilon)$ such that

$$
\stackrel{0}{z, \delta} V_{\eta}(M)+\epsilon / \delta^{2} \geqslant{ }_{y 0} V_{\eta}(M, L) \text { for } L \geqslant L_{2}^{1}(\delta, \epsilon), M \geqslant 0, \eta>0 .
$$

(vii) There exists $M_{1}^{1}(\delta, \epsilon)$ such that

$$
{ }_{z 0} V^{\eta}(M, L) \geqslant \underset{y, \delta}{0} V^{\eta}(L)-\epsilon / \delta^{2} \quad \text { for } M \geqslant M_{1}^{1}(\delta, \epsilon), L \geqslant 0, \eta>0 .
$$

(viii) There exists $M_{2}^{1}(\delta, \epsilon)$ such that

$$
{ }_{z 0} V_{\eta}(M, L) \geqslant{ }_{y, \delta}^{0} V_{\eta}(L)-\epsilon / \delta^{2} \quad \text { for } M \geqslant M_{2}^{1}(\delta, \epsilon), L \geqslant 0, \eta>0 .
$$

Proof. (i) Fix $\delta>0$. Since

$$
{ }_{z, \delta} V^{\eta}=\inf _{z, \delta} \sup _{y} P\left[{ }^{z, \delta} \Delta_{\eta}(y), y\right],
$$

given $\epsilon>0$, there exists a lower $\eta$-strategy ${ }^{z, \delta} \tilde{\Delta}_{\eta}$ for $z$ such that

$$
{ }_{2, \delta} V^{\eta} \geqslant P\left[{ }^{2, \delta} \widetilde{\Delta}_{\eta}(y), y\right]-\epsilon / 2
$$

for all measurable controls $y$.

Given any control $y$, let $y$ have components $y=\left(y_{1}, \ldots, y_{\beta}\right)$. Let $\left(\tilde{z}_{\eta}, y\right)$ denote the outcome of $\left({ }^{z, \delta} \tilde{\Delta}_{\eta}(y), y\right)$. Then $\tilde{z}_{\eta}$ is constant on $\delta$-squares and $y$ is measurable. Let $\tilde{z}_{\eta}$ have components $\tilde{z}_{\eta}=\left(\tilde{z}_{1}, \ldots, \tilde{z}_{\beta}\right)$ where $\tilde{z}_{1}={ }^{z, \delta} \tilde{\Delta}_{\eta, 1}, \quad \tilde{z}_{j}={ }^{z, \delta} \tilde{\Delta}_{\eta, j}\left(\tilde{z}_{1}, y_{1}, \tilde{z}_{2}, y_{2}, \ldots, \tilde{z}_{j-1}, y_{j-1}\right) \quad(2 \leqslant j \leqslant \beta)$. Assume without loss of generality that $\eta \leqslant \delta$.

We can define a lower $\eta$-(L)-strategy, $L \tilde{\Delta}_{\eta}$, for $z$ with the following property:

Given any measurable control $y$, the $z$-outcome of $\left({ }^{L} \tilde{\Delta}_{\eta}(y), y\right)$ denoted by $\left(y, \tilde{z}_{L, \eta}\right)$ satisfies

$$
\left\|\tilde{z}_{L, \eta}-\tilde{z}_{\eta}\right\|_{L^{1}}<\sigma(L)<\epsilon / \delta^{2} \text { when } L \geqslant L_{1}(\delta, \epsilon)
$$

where $\sigma(r)$ is a positive function, $\sigma(r) \rightarrow 0$ as $r \rightarrow \infty$.

Indeed, we can define ${ }^{L} \tilde{\Delta}_{\eta}$ such that $\tilde{z}_{L, \eta}$ agrees with $\tilde{z}_{\eta}$ for every $\eta$-interval not containing points of the $\delta$-partition. For those $\eta$-intervals which do contain points of the $\delta$-partition, $\widetilde{z}_{L, \eta}$ is the linear function joining the constants of $\tilde{z}_{\eta}$ within a distance $\epsilon$. We do this in both $t$ and $x$. Thus, at each $\delta$-point we introduce an error term of $C \epsilon / \delta^{2}$ and $\widetilde{z}_{L, \eta}$ will be a member of $Z^{L}([0, T] \times[0, B])$ 
with $L \geqslant L_{1}(\delta, \epsilon) \geqslant C \epsilon / \delta^{2}$ ( $C$ is a constant depending only on the control $\operatorname{set} Z$ ).

By the continuity of $h$ it then follows from (2.15) that for all measurable controls $y$

$$
\left|P\left(\tilde{z}_{L, \eta}, y\right)-P\left(\tilde{z}_{\eta}, y\right)\right|<\epsilon / 2 \delta^{2} \quad \text { when } L \geqslant L_{1}(\delta, \epsilon)
$$

so

$$
P\left(\tilde{z}_{\eta}, y\right) \geqslant P\left(\tilde{z}_{L, \eta} y\right)-\epsilon / 2 \delta^{2} \quad\left(L \geqslant L_{1}(\delta, \epsilon)\right)
$$

and so using (2.14), (i) follows. The proofs of (ii)-(viii) are similar.

LEMMA 2.4. For each $\epsilon>0$ and for each $L \geqslant 0, M \geqslant 0$

(i) There exists $\delta_{1}(L, \epsilon)$ such that

$$
V^{\eta}(L){ }_{2, \delta} V^{\eta}-\epsilon \text { for } \delta \leqslant \delta_{1}(L, \epsilon), \eta>0 .
$$

(ii) There exists $\delta_{2}(L, \epsilon)$ such that

$$
V_{\eta}(L){ }_{2, \delta} V_{\eta}-\epsilon \text { for } \delta \leqslant \delta_{2}(L, \epsilon), \eta>0 .
$$

(iii) There exists $\delta_{3}(M, \epsilon)$ such that

$$
V^{\eta}(M) \leqslant{ }_{y, \delta} V^{\eta}+\epsilon \text { for } \delta \leqslant \delta_{3}(M, \epsilon), \eta>0 .
$$

(iv) There exists $\delta_{4}(M, \epsilon)$ such that

$$
V_{\eta}(M) \leqslant{ }_{y, \delta} V_{\eta}+\epsilon \text { for } \delta \leqslant \delta_{4}(M, \epsilon), \eta>0
$$

(v) There exists $\delta_{1}^{1}(L, \epsilon)$ such that

$$
{ }_{y_{0}} V^{\eta}(M, L) \geqslant \stackrel{0}{{ }_{z, \delta}} V^{\eta}(M)-\epsilon \text { for } \delta \leqslant \delta_{1}^{1}(L, \epsilon), M \geqslant 0, \eta>0 .
$$

(vi) There exists $\delta_{2}^{1}(L, \epsilon)$ such that

$$
{ }_{y 0} V_{\eta}(M, L) \geqslant{ }_{z, \delta} \stackrel{0}{V}_{\eta}(M)-\epsilon \text { for } \delta \leqslant \delta_{2}^{1}(L, \epsilon), M \geqslant 0, \eta>0 .
$$

(vii) There exists $\delta_{3}^{1}(M, \epsilon)$ such that

$$
{ }_{z 0} V^{\eta}(M, L) \leqslant \stackrel{0}{0} V_{y, \delta}^{\eta}(L)+\epsilon \text { for } \delta \leqslant \delta_{3}^{1}(M, \epsilon), L \geqslant 0, \eta>0 .
$$

(viii) There exists $\delta_{4}^{1}(M, \epsilon)$ such that

$$
{ }_{20} V_{\eta}(M, L) \leqslant \stackrel{0}{0} \stackrel{y}{V}, \delta_{\eta}(L)+\epsilon \text { for } \delta \leqslant \delta_{4}^{1}(M, \epsilon), L \geqslant 0, \eta>0 .
$$

Proof. (i) Let $\epsilon>0$ be given and fix $L \geqslant 0$. Since for fixed $L$, $Z^{L}([0, T] \times[0, B])$ is compact (in the topology of continuous functions or $\left.L^{1}\right)$, there exists a partition $\pi$ of $[0, T] \times[0, B]$ such that given $z_{L} \in Z^{L}$, there is 
a function $z_{\pi}$ which is a constant on each rectangle of the $\pi$ partition with $\left\|z_{L}-z_{\pi}\right\|<\epsilon$. Note that $\pi$ is independent of which $z_{L} \in Z^{L}$ we choose and depends only on $L$ and $\epsilon$.

Choose $\alpha_{1}=\alpha_{1}(L, \epsilon)$ such that if we partition $[0, T] \times[0, B]$ into $\delta_{1}(L, \epsilon)=T / \alpha_{1}$ squares, then the $\delta$-partition will be finer than the $\pi$-partition. Thus, given $z_{L} \in Z^{L}$, there exists a function $z$, constant on $\delta$-squares, for all $\delta \leqslant$ $\delta_{1}(L, \epsilon)$ with $\left\|z-z_{L}\right\|_{L^{1}}<\epsilon$.

Now, since

$$
V^{\eta}(L)=\inf _{L_{\Delta_{\eta}}} \sup _{y} P\left[{ }^{L} \Delta_{\eta}(y), y\right]
$$

there exists a lower $\eta$-(L)-strategy $L \widetilde{\Delta}_{\eta}$ for $z$ such that

$$
V^{\eta}(L) \geqslant P\left[{ }^{L} \tilde{\Delta}_{\eta}(y), y\right]-\epsilon
$$

for all measurable controls $y$.

With the above $\delta_{1}(L, \epsilon)$ it is easy to see that we can define a lower $\eta$-strategy $z, \delta \widetilde{\Delta}_{\eta}$ for $z$ with the following property:

Given any measurable control $y$, the $z$-outcome of $\left({ }^{z, \delta} \widetilde{\Delta}_{\eta}(y), y\right)$ denoted by $\left(\tilde{z}_{\eta}, y\right)$ satisfies

$$
\left\|\tilde{z}_{L, \eta}-\tilde{z}_{\eta}\right\|_{L^{1}}<\epsilon \text { when } \delta<\delta_{1}(L, \epsilon)
$$

where $\widetilde{z}_{L, \eta}$ is the $z$-outcome of $\left({ }^{L} \widetilde{\Delta}_{\eta}(y), y\right)$. Here $\tilde{z}_{\eta}$ will be a function which is a constant on $\delta$-squares.

By the continuity of $h$ and by (2.17) there is a positive function $\sigma(r), \sigma(r)$ $\rightarrow 0$ as $r \rightarrow 0$ such that for all measurable controls $y$

$$
\left|P\left(\tilde{z}_{\eta}, y\right)-P\left(\tilde{z}_{L, \eta} y\right)\right| \leqslant \sigma(\delta)<\epsilon \text { when } \delta \leqslant \delta_{1}(L, \epsilon) .
$$

Combining this with (2.16) yields (i). Similarly (ii)-(viii) are shown.

THEOREM 2.2. Let (A), (B) hold. Then

(i) $\quad \lim _{\delta \rightarrow 0} V^{\delta}$ exists, say $\lim _{\delta \rightarrow 0} V^{\delta}={ }_{z} V^{+}$and $\lim _{L \rightarrow \infty} V^{+}(L)={ }_{z} V^{+}$,

(ii) $\lim _{L \rightarrow \infty} V^{-}(L)={ }_{2} V^{+}$,

(iii) $\quad \lim _{\delta \rightarrow 0}{ }_{y} V_{\delta}$ exists, say $\lim _{\delta \rightarrow 0}{ }_{y} V_{\delta}={ }_{y} V^{-}$and $\lim _{M \rightarrow \infty} V^{+}(M)={ }_{y} V^{-}$,

(iv) $\lim _{M \rightarrow \infty} V^{-}(M)={ }_{y} V^{-}$, 
(v) $\quad \lim _{\delta \rightarrow 0} z_{z}{ }^{\delta} \delta(M)$ exists for each $M$, say $\lim _{\delta \rightarrow 0}{ }_{2}{ }^{0} \delta(M)={ }_{z} V^{+}(M)$, and $\lim _{L \rightarrow \infty}{ }_{y 0} V^{+}(M, L)={ }_{2} V^{+}(M)$ for each $M \geqslant 0$,

(vi) $\quad \lim _{L \rightarrow \infty}{ }{ } V^{-}(M, L)={ }_{2} V^{+}(M)$ for each $M \geqslant 0$,

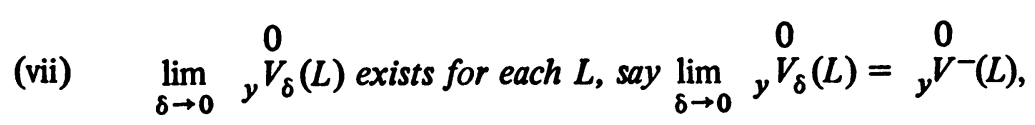
and $\lim _{M \rightarrow \infty}{ }_{20} V^{+}(M, L)={ }_{y} V^{-}(L)$ for each $L \geqslant 0$,

(viii) $\lim _{M \rightarrow \infty} 20^{-}(M, L)={ }_{y} V^{-}(L)$ for each $L \geqslant 0$.

Proof. (i) Given $\epsilon^{1}>0$ and $\delta>0$, let $\epsilon=\delta^{2} \epsilon^{1}$. Then by Lemma 2.3(i) there exists $L_{1}(\delta, \epsilon)$ such that

$$
z, \delta V^{\eta}+\epsilon \geqslant V^{\eta}(L) \text { for } L \geqslant L_{1}(\delta, \epsilon), \eta>0 .
$$

In particular

$$
z, \delta V^{\eta}+\epsilon \geqslant V^{\eta}\left(L_{1}(\delta, \epsilon)\right) \text { for all } \eta>0 .
$$

Given this $L_{1}(\delta, \epsilon)$, by Lemma 2.4(i) there exists $\delta_{1}\left(L_{1}, \epsilon\right)$ such that

$$
V^{\eta}\left(L_{1}(\delta, \epsilon)\right) \geqslant_{2, \delta} V^{\eta}-\epsilon \text { for } \delta \leqslant \delta_{1}\left(L_{1}, \epsilon\right), \eta>0 .
$$

In particular

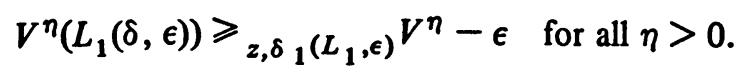

Combining (2.18), (2.19) we obtain

$$
{ }_{2, \delta} V^{\eta}+\epsilon \geqslant V^{\eta}\left(L_{1}(\delta, \epsilon)\right) \geqslant_{2, \delta_{1}\left(L_{1}, \epsilon\right)} V^{\eta}-\epsilon \text { for all } \eta>0 .
$$

Letting $\eta \rightarrow 0$ in (2.20) and using Lemma 2.2(i) and Remark 2.2 we obtain

$$
z V^{\delta}+\epsilon \geqslant V^{+}\left(L_{1}(\delta, \epsilon)\right) \geqslant V_{2} \delta_{1}\left(L_{1}, \epsilon\right)-\epsilon .
$$

Let $\delta \rightarrow 0$. Then $L_{1}(\delta, \epsilon) \rightarrow \infty$ and $\delta_{1}\left(L_{1}, \epsilon\right)=\delta_{1}(\delta, \epsilon) \rightarrow 0$ so that (2.21) implies

$$
\liminf _{\delta \rightarrow 0} V^{\delta} \geqslant \lim _{L \rightarrow \infty} V^{+}(L) \geqslant \limsup _{\delta \rightarrow 0} V^{\delta}
$$


As remarked before, $\lim _{L \rightarrow \infty} V^{+}(L)$ exists since $V^{+}(L)$ is monotone decreasing in $L ; \lim _{L \rightarrow \infty} V^{+}(L)=\inf _{L} V^{+}(L)$. Thus

$$
\lim _{\delta \rightarrow 0} V^{\delta} \text { exists and } \lim _{\delta \rightarrow 0} V^{\delta}={ }_{z} V^{+}=\lim _{L \rightarrow \infty} V^{+}(L) \text {. }
$$

(ii) Given $\epsilon^{1}>0$ and $\delta>0$, let $\epsilon=\delta^{2} \epsilon^{1}$. Then, by Lemma 2.3(ii) there exists $L_{2}(\delta, \epsilon)$ such that

$$
{ }_{2, \delta} V_{\eta}+\epsilon \geqslant V_{\eta}(L) \text { for } L \geqslant L_{2}(\delta, \epsilon), \eta>0 .
$$

In particular

$$
{ }_{2, \delta} V_{\eta}+\epsilon \geqslant V_{\eta}\left(L_{2}(\delta, \epsilon)\right) \text { for all } \eta>0 .
$$

Given this $L_{2}(\delta, \epsilon)$, by Lemma $2.4(i i)$, there exists $\delta_{2}\left(L_{2}, \epsilon\right)$ such that

$$
V_{\eta}\left(L_{2}(\delta, \epsilon)\right) \geqslant_{2, \delta} V_{\eta}-\epsilon \text { for } \delta \leqslant \delta_{2}\left(L_{2}, \epsilon\right), \eta>0 .
$$

In particular

$$
V_{\eta}\left(L_{2}(\delta, \epsilon)\right) \geqslant_{2, \delta}\left(L_{2}, \epsilon\right) V_{\eta}-\epsilon \text { for all } \eta>0 .
$$

Combining (2.22), (2.23) we get

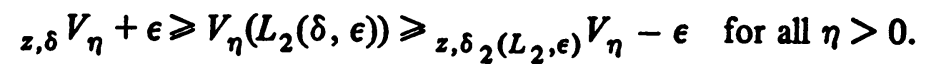

Letting $\eta \rightarrow 0$ and using Lemma 2.2(ii) and Remark 2.2 we obtain from (2.24)

$$
{ }_{2} V^{\delta}+\epsilon \geqslant V^{-}\left(L_{2}(\delta, \epsilon)\right)>{ }_{2} V^{\delta}{ }_{2}\left(L_{2}, \epsilon\right)-\epsilon .
$$

If we let $\delta \rightarrow 0$, then $L_{2}(\delta, \epsilon) \rightarrow \infty$ and $\delta_{2}\left(L_{2}, \epsilon\right)=\delta_{2}(\delta, \epsilon) \rightarrow 0$. Then (2.25) yields using part (i)

$$
{ }_{2} V^{+}>\lim _{L \rightarrow \infty} V^{-}(L) \geqslant{ }_{2} V^{+} \text {. }
$$

Thus ${ }_{2} V^{+}=\lim _{L \rightarrow \infty} V^{-}(L)=\inf _{L} V^{-}(L)$. Similarly we prove (iii)-(viii).

We introduce one final notion of value.

Given a positive integer $\gamma$, let $\mu=T / \gamma$ and partition $[0, T] \times[0, B]$ into $\mu$-squares:

$$
J_{j} \times J_{k}=\left\{(t, x) \in[0, T] \times[0, B]: \mu_{j-1}<t \leqslant \mu_{j}, \mu_{k-1}<x \leqslant \mu_{k}\right\}
$$

for $1 \leqslant j, k \leqslant \gamma$, where $\mu_{j}=j \cdot \mu, \mu_{0}=0, \mu_{\gamma}=T=B$. We call $J_{j} \times J_{k}$ a $\mu$-square.

Definition. Let $\underset{\substack{y, \mu \\ z, \delta}}{ } V^{\eta}$ denote the upper $\eta$-value with $z$ choosing control functions constant on $\delta$-squares and $y$ choosing control functions constant on $\mu$-squares. The corresponding $\eta$-strategies are denoted by ${ }^{2, \delta} \Delta_{\eta},{ }^{y, \mu} \Gamma^{\eta}$.

Similarly we define $\underset{z, \delta}{y, \mu} V_{\eta}$. 
LEMMA 2.5. For each $\epsilon>0$ and for each $L \geqslant 0, M \geqslant 0$,

(i) There exists $\delta_{1}(L, \epsilon)$ such that

$$
\underset{y, \mu}{\stackrel{0}{V}{ }^{\eta}(L)} \geqslant_{\substack{y, \mu \\ z, \delta}} V^{\eta}-\epsilon \text { for } \delta \leqslant \delta_{1}(L, \epsilon), \mu, \eta>0 .
$$

(ii) There exists $\delta_{2}(L, \epsilon)$ such that

$$
\stackrel{0}{y, \mu}_{\eta}(L) \geqslant \underset{\substack{y, \mu \\ z, \delta}}{ } V_{\eta}-\epsilon \text { for } \delta \leqslant \delta_{2}(L, \epsilon), \mu, \eta>0 .
$$

(iii) There exists $\delta_{3}(M, \epsilon)$ such that

$$
\stackrel{0}{V_{z, \mu}^{\eta}}(M) \leqslant_{\substack{z, \mu \\ y, \delta}} V^{\eta}+\epsilon \text { for } \delta \leqslant \delta_{3}(M, \epsilon), \mu, \eta>0 .
$$

(iv) There exists $\delta_{4}(M, \epsilon)$ such that

$$
\stackrel{0}{V}, \mu_{\eta}(M) \leqslant \underset{\substack{z, \mu \\ y, \delta}}{ } V_{\eta}+\epsilon \text { for } \delta \leqslant \delta_{4}(M, \epsilon), \mu, \eta>0 .
$$

The proof of Lemma 2.5 is similar to that of Lemma 2.8 and is omitted.

LEMMA 2.6.

(i)

$$
\lim _{L \rightarrow \infty} V^{+}(L)=\lim _{L \rightarrow \infty} \stackrel{0}{V}^{+}(L),
$$

(ii)

$$
\lim _{L \rightarrow \infty} V^{-}(L)=\lim _{L \rightarrow \infty}{\stackrel{0}{V^{-}}}^{-}(L)
$$

$$
\lim _{M \rightarrow \infty} V^{+}(M)=\lim _{M \rightarrow \infty} \stackrel{0}{V}^{+}(M)
$$

$$
\lim _{M \rightarrow \infty} V^{-}(M)=\lim _{M \rightarrow \infty} \stackrel{0}{V}^{-}(M),
$$

(v)

$$
\lim _{M \rightarrow \infty} \lim _{L \rightarrow \infty} y_{0} V^{+}(M, L)=\lim _{M \rightarrow \infty} \lim _{L \rightarrow \infty} V^{+}(M, L),
$$

$$
\lim _{M \rightarrow \infty} \lim _{L \rightarrow \infty} y_{0} V^{-}(M, L)=\lim _{M \rightarrow \infty} \lim _{L \rightarrow \infty} V^{-}(M, L),
$$

$$
\lim _{L \rightarrow \infty} \lim _{M \rightarrow \infty} 20 V^{+}(M, L)=\lim _{L \rightarrow \infty} \lim _{M \rightarrow \infty} V^{+}(M, L),
$$

$$
\lim _{L \rightarrow \infty} \lim _{M \rightarrow \infty} z 0^{-}(M, L)=\lim _{L \rightarrow \infty} \lim _{M \rightarrow \infty} V^{-}(M, L) .
$$


Proof. (i) Since $V^{+}(L) \leqslant \stackrel{0}{V}^{+}(L)$ for every $L$,

$$
\lim _{L \rightarrow \infty} V^{+}(L) \leqslant \lim _{L \rightarrow \infty}{\stackrel{0}{V^{+}}}^{+}(L)
$$

we need only show

$$
\lim _{L \rightarrow \infty}{\stackrel{0}{V^{+}}}^{+}(L) \leqslant \lim _{L \rightarrow \infty} V^{+}(L)
$$

Since

$$
V^{\delta}(L)=\inf _{L_{\Delta_{\delta}}} \sup _{y} P\left[{ }^{L} \Delta_{\delta}(y), y\right]
$$

given $\epsilon>0$, there is a lower $\delta-(L)$-strategy ${ }^{L} \tilde{\Delta}_{\delta}$ such that

$$
V^{\delta}(L) \geqslant P\left[{ }^{L} \tilde{\Delta}_{\delta}(y), y\right]-\epsilon
$$

for all measurable controls $y$.

Let $\left(\tilde{z}_{L, \delta}, y\right)$ denote the outcome of $\left({ }^{L} \tilde{\Delta}_{\delta}(y), y\right)$ given a control $y$ with components $y=\left(y_{1}, \ldots, y_{\alpha}\right)$. Suppose $\tilde{z}_{L, \delta}$ has components $\tilde{z}_{L, \delta}=$ $\left(\tilde{z}_{L, 1}, \ldots, \tilde{z}_{L, \alpha}\right)$. Define the lower $\delta$ - $\left(L_{0}\right)$-strategy for $z$ as follows for some $L_{0}$ to be determined.

Let $\bar{z} \in Z$ denote the fixed element such that $z_{L}(0, x)=\bar{z}$ for all $z_{L} \in$ $Z_{0}^{L}([0, T] \times[0, B])$. Let

$$
L_{0}^{0} \stackrel{0}{\tilde{\Delta}_{\delta, 1}}=\tilde{z}_{L_{0}, 1}
$$

0

where $\tilde{z}_{L_{0}, 1}$ is the function defined on $\left[0, \delta_{1}\right] \times[0, B]$ obtained by joining $\bar{z}$ to $\widetilde{z}_{L, 1}={ }_{L} \widetilde{\Delta}_{\delta, 1}$ linearly within a distance $\epsilon$ of the $t$-axis.

Let $z$ play the same linear function in every $\delta$-interval until it meets $\tilde{z}_{L, \delta}$. Assume without loss of generality the linear function meets $\tilde{z}_{L, \delta}$ in the first $\delta$-interval. Then let

$$
\left.\stackrel{0}{L_{0}} \underset{\tilde{\Delta}_{\delta, 2}}{\stackrel{0}{\tilde{z}_{L_{0}, 1}}}, y_{1}\right)={\stackrel{0}{z_{L_{0}, 2}}}^{0} \tilde{z}_{L, 2}
$$

and, for $3 \leqslant j \leqslant \alpha$

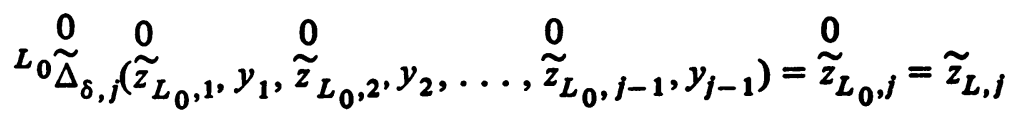

where

$$
\tilde{z}_{L, j}={ }^{L} \tilde{\Delta}_{\delta, j}\left(\tilde{z}_{L, 1}, y_{1}, \ldots, \tilde{z}_{L, j-1}, y_{j-1}\right)
$$




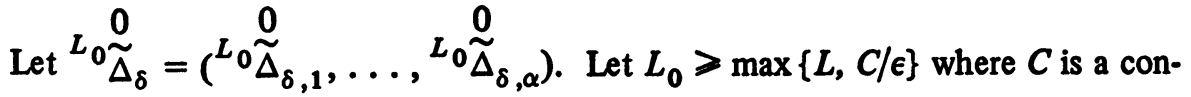
stant bounding the control set $Z$. Then

$$
{\stackrel{0}{\tilde{z}_{L_{0}, \delta}}}=\left({\stackrel{\tilde{z}}{L_{0}, 1}}_{1}, \ldots,{\stackrel{0}{\tilde{z}_{L_{0}, \alpha}}}\right) \in Z_{0}^{L_{0}}([0, T] \times[0, B]) .
$$

Also it is clear that there is a positive function $\sigma(r), \sigma(r) \rightarrow 0$ as $r \rightarrow 0$ such that $\left\|\widetilde{z}_{L_{0}, \delta}-\tilde{z}_{L, \delta}\right\|_{L^{1}} \leqslant \sigma(\epsilon)$. Thus, by the continuity of $h$, there is another positive function $\sigma$ going to zero with its argument such that for every measurable control $y$

$$
\left|P\left(y, \tilde{z}_{L_{0}, \delta}\right)-P\left(y, \tilde{z}_{L, \delta}\right)\right| \leqslant \sigma(\epsilon) .
$$

Combining (2.27), (2.28) and using the fact that

$$
\stackrel{0}{V}^{\delta}(L)=\inf _{\substack{L_{\Delta_{\delta}}^{0} \\ \sup _{y}}} P\left[{ }^{L}{ }_{\Delta_{\delta}}^{0}(y), y\right]
$$

we obtain

$$
V^{\delta}(L) \geqslant \stackrel{0}{V^{\delta}}\left(L_{0}\right)-\epsilon-\sigma(\epsilon) .
$$

Since $\sigma$ is independent of $\delta$

$$
V^{+}(L) \geqslant \stackrel{0}{V}^{+}\left(L_{0}\right)-\epsilon-\sigma(\epsilon) .
$$

Hence

$$
\lim _{L \rightarrow \infty} V^{+}(L) \geqslant \lim _{L \rightarrow \infty} \stackrel{0}{V^{+}}(L)
$$

Similarly we prove (ii)-(viii).

THEOREM 2.3. Let (A), (B) hold. Then
(i) $\lim _{M \rightarrow \infty}{ }_{2} V^{+}(M)={ }_{y} V^{-}$,
(ii) $\lim _{L \rightarrow \infty}{ }_{y^{V}} \stackrel{0}{-}^{-}(L)={ }_{2} V^{+}$.

Proof. We will only prove (ii); (i) is similar. By Theorem 2.2 and Lemma $2.6 \lim _{L \rightarrow \infty} V^{+}(L)=\lim _{L \rightarrow \infty} \stackrel{0}{V}^{+}(L)={ }_{2} V^{+}$.

Thus, given $\epsilon>0$, there is $L_{0}(\epsilon)$ such that

$$
{ }_{2} V^{+}+\epsilon \geqslant \stackrel{0}{V^{+}}(L) \text { for } L \geqslant L_{0}(\epsilon) .
$$

Since $\stackrel{0}{V}^{+}(L) \geqslant \stackrel{0}{V}^{-}(L) \geqslant \stackrel{0}{V}^{-}(L)$ for all $L \geqslant 0$ we have by (2.29) 


$$
{ }_{z} V^{+}+\epsilon \geqslant \stackrel{0}{V^{-}}(L) \text { for } L \geqslant L_{0}(\epsilon)
$$

That is

$$
\lim _{L \rightarrow \infty} \stackrel{0}{y^{-}}(L) \leqslant{ }_{z} V^{+} .
$$

Note that $\lim _{L \rightarrow \infty} \stackrel{\text { y }}{V}^{-}(L)$ exists by monotonicity. Thus we have left to show

$$
{ }_{y} V^{-}(L)+\epsilon \geqslant{ }_{z} V^{+} \text {for } L \text { sufficiently large. }
$$

Since

$$
z, \delta V^{\eta}=\inf _{z} \sup _{\Gamma^{\eta}} P\left[z, \Gamma^{\eta}(z)\right]
$$

there exists an upper $\eta$-strategy $\widetilde{\Gamma}^{\eta}$ for $y$ such that

$$
{ }_{z, \delta} V^{\eta} \leqslant P\left[z, \widetilde{\Gamma}^{\eta}(z)\right]+\epsilon
$$

for all controls $z$ constant on $\delta$-squares.

Recall that $\eta=T / \beta, \delta=T / \alpha$. For convenience and without loss of generality we choose $\alpha, \beta$ such that $\alpha$ divides $\beta$.

Let $Q_{\delta}=$ \{class of all controls $z$ which are constants on $\delta$-squares with values in $Z\}$. Then $Q_{\delta}$ is a component subject of $L^{1}([0, T] \times[0, B])$ (being finite dimensional).

Thus, there are a finite number of control functions $z^{1}, \ldots, z^{s} \in Q_{\delta}$ such that given any $z \in Q_{\delta}$ there is an index $i, 1 \leqslant i \leqslant s$, such that $\left\|z-z^{i}\right\|_{L^{1}}<\epsilon$. Note that the $z^{i}, 1 \leqslant i \leqslant s$, can be chosen so that they coincide for no $(t, x) \in$ $[0, T] \times[0, B]$. Note also that $s=s(\delta, \epsilon)$.

Define the following upper $\eta$-strategy $\widetilde{\widetilde{\Gamma}}^{\eta}$ for $y$ :

Let $z \in Q_{\delta}$ be given having components $z=\left(z_{1}, \ldots, z_{\beta}\right)$. Let

$$
\widetilde{\Gamma}^{\eta, 1}\left(z_{1}\right)=\widetilde{\Gamma}^{\eta, 1}\left(\left.z^{i}\right|_{K_{1} \times[0, B]}\right)=\tilde{y}_{1}^{i}
$$

where $\left\|z_{1}-\left.z^{i}\right|_{K_{1} \times[0, B]}\right\|_{L_{1}} \leqslant \epsilon, K_{1}=\left\{t \in[0, T]: \eta_{0}=0 \leqslant t \leqslant \eta_{1}\right\}, \tilde{y}_{1}^{i}=$ $\tilde{\Gamma}^{\eta, 1}\left(\left.z^{i}\right|_{K_{1} \times[0, B]}\right)$ for each $z_{1}$, with $\left\|z_{1}-\left.z^{i}\right|_{K_{1} \times[0, B]}\right\|_{L_{1}}<\epsilon$.

$$
\widetilde{\Gamma}^{\eta, 2}\left(z_{1}, \tilde{y}_{1}^{i}, z_{2}\right)=\tilde{\Gamma}^{\eta, 2}\left(\left.z^{i}\right|_{K_{1} \times[0, B]}, \tilde{y}_{1}^{i},\left.z^{i}\right|_{K_{2} \times[0, B]}\right)=\tilde{y}_{2}^{i}
$$

where $K_{2}=\left\{t \in[0, T]: \eta_{1}<t \leqslant \eta_{2}\right\}$,

$$
\tilde{y}_{2}^{i}=\tilde{\Gamma}^{\eta, 2}\left(\left.z^{i}\right|_{K_{1} \times[0, B]}, \tilde{y}_{1}^{i},\left.z^{i}\right|_{K_{2} \times[0, B]}\right) \text { for each } z_{2} \text {. }
$$

In general we continue in this fashion until we reach the $\eta$-subinterval say $K_{l}=\left\{t \in[0, T]: \eta_{l-1}<t \leqslant \eta_{l}\right\}$ such that $\eta_{l}=\delta_{1}$. In $K_{l}$ 


$$
\begin{aligned}
& \widetilde{\Gamma}^{\eta, l}\left(z_{1}, \tilde{y}_{1}^{i}, z_{2}, \tilde{y}_{2}^{i}, \ldots, \tilde{y}_{l-1}^{i}, z_{l}\right) \\
& =\widetilde{\Gamma}^{\eta, l}\left(\left.z^{i}\right|_{K_{1} \times[0, B]}, \tilde{y}_{1}^{i}, \ldots,\left.z^{i}\right|_{K_{l-1} \times[0, B]}, \tilde{y}_{l-1}^{i},\left.z^{i}\right|_{K_{l} \times[0, B]}\right)=\tilde{y}_{l}^{i} .
\end{aligned}
$$

In $K_{l+1}=\left\{t \in[0, T]: \eta_{l}<t \leqslant \eta_{l+1}\right\}$

$$
\begin{aligned}
& \tilde{\Gamma}^{\eta, l+1}\left(z_{1}, \tilde{y}_{1}^{i}, z_{2}, \tilde{y}_{2}^{i}, \ldots, z_{l-1}, \tilde{y}_{l-1}^{i}, z_{l}, \tilde{y}_{l}^{i}, z_{l+1}\right) \\
& \quad=\tilde{\Gamma}^{\eta, l+1}\left(\left.z^{i}\right|_{K_{1} \times[0, B]}, \tilde{y}_{1}^{i}, \ldots,\left.z^{i}\right|_{K_{l} \times[0, B]}, \tilde{y}_{l}^{i},\left.z^{k}\right|_{K_{l+1} \times[0, B]}\right)=\tilde{y}_{l+1}^{k}
\end{aligned}
$$

where $\left\|z_{l+1}-\left.z^{k}\right|_{K_{l+1}} \times[0, B]\right\|_{L}<\epsilon$,

$$
\tilde{y}_{l+1}^{k}=\tilde{\Gamma}^{\eta, l+1}\left(\left.z^{i}\right|_{K_{1} \times[0, B]}, \tilde{y}_{1}^{i}, \ldots,\left.z^{i}\right|_{K_{l} \times[0, B]}, \tilde{y}_{l}^{i},\left.z^{k}\right|_{K_{l+1} \times[0, B]}\right)
$$

for each $z_{l+1}$ with

$$
\left\|z_{l+1}-\left.z^{k}\right|_{K_{l+1} \times[0, B]}\right\|_{L^{1}}<\epsilon .
$$

Proceed as above up to completion of the game. Define

$$
\widetilde{\Gamma}^{\eta}=\left(\widetilde{\Gamma}^{\eta, 1}, \ldots, \widetilde{\Gamma}^{\eta, \beta}\right) \text {. }
$$

Then $\widetilde{\Gamma}^{\eta}$ is defined on all of $Q_{\delta}$ and since there are $\alpha \delta$-partition points $\delta_{0}=0$, $\delta_{1}, \ldots, \delta_{\alpha-1}$ in $[0, T]$, by the definition of $\widetilde{\Gamma}^{\eta}$ there are at most $s^{\alpha} y$-responses for every $z \in Q_{\delta}$. If we let

$$
\begin{aligned}
\tilde{z}=\left(\left.z^{i}\right|_{K_{1} \times[0, B]},\left.z^{i}\right|_{K_{2} \times[0, B]}, \ldots,\left.z^{i}\right|_{K_{l} \times[0, B]},\right. \\
\left.\left.z^{k}\right|_{K_{l+1} \times[0, B]}, \ldots,\left.z^{p}\right|_{K_{\beta} \times[0, B]}\right)
\end{aligned}
$$

then, by the way in which $\tilde{z}$ was defined it is clear that $\tilde{z} \in Q_{\delta}$ and

$$
\|z-\tilde{z}\|_{L^{1}}<\epsilon .
$$

Note that for each $z \in Q_{\delta}$, the $y$-outcome of $\left(z, \widetilde{\Gamma}^{\eta}(z)\right)$ is a measurable function.

Since there are at most $s^{\alpha} y$-responses of $\widetilde{\Gamma}^{\eta}$ against any control $z \in Q_{\delta}$ there is a positive integer $\gamma=\gamma(\delta, \epsilon)$ such that we can modify $\widetilde{\widetilde{\Gamma}}^{\eta}$ into an $\eta$-strategy ${ }^{y, \mu} \widetilde{\Gamma}^{\eta}$, where $\mu=\mu(\delta, \epsilon)=T / \gamma$, having the property that given any control $z \in Q_{\delta}$

$$
\left\|\widetilde{\tilde{y}}^{\eta}-\widetilde{\tilde{y}}^{\mu}\right\|_{L^{1}}<\epsilon
$$

where $\widetilde{y}^{\eta}$ is the $y$-outcome of $\left(z, \widetilde{\Gamma}^{\eta}(z)\right), \widetilde{\widetilde{y}}^{\mu}$ is the $y$-outcome of $\left(z,,^{y, \mu} \widetilde{\Gamma}^{\eta}(z)\right)$ and $\widetilde{\tilde{y}}^{\mu}$ is constant on $\mu$-squares. Indeed the modification is similar to that carried out in Lemma 2.4. Note that $\mu$ is independent of $\eta$. 
Now, (2.32) and (2.33) imply that the corresponding trajectories are within $\epsilon$ of each other. Thus combining (2.31), (2.32), (2.33) and using the continuity of $h$ we obtain

$$
z, \delta V^{\eta} \leqslant \underset{\substack{y, \mu \\ z, \delta}}{ } V^{\eta}+\sigma(\epsilon)
$$

where $\sigma$ is a positive function going to zero with its argument and is independent of $\eta, \mu, \delta$.

Now, by Lemma $2.5(i)$, for each $L \geqslant 0$, there exists a $\delta_{1}(L, \epsilon)>0$ such that

$$
\underset{y, \mu}{V^{\eta}}(L) \geqslant \underset{\substack{y, \mu \\ z, \delta}}{ } V^{\eta}-\epsilon
$$

when $\delta \leqslant \delta_{1}(L, \epsilon), \eta>0, \mu=\mu(\delta, \epsilon)$. Combining (2.34), (2.35) yields

$$
{ }_{z, \delta} V^{\eta} \leqslant \underset{y, \mu}{0} V^{\eta}(L)+\sigma(\epsilon), \quad \delta \leqslant \delta_{1}(L, \epsilon), \mu=\mu(\delta, \epsilon), \eta>0,
$$

for another function $\sigma$ as above.

Let $\eta \rightarrow 0$ in (2.36) and use Lemma 2.2(i), (vii) to obtain

$$
{ }_{z} V^{\delta} \leqslant{ }_{y} \stackrel{0}{\mu}_{\mu}(L)+\sigma(\epsilon), \quad \delta \leqslant \delta_{1}(L, \epsilon), \mu=\mu(\delta, \epsilon) .
$$

Let (i) $\delta \rightarrow 0$, (ii) $\mu \rightarrow 0$, (iii) $L \rightarrow \infty$ in (2.37). Thus, using Theorem 2.2

$$
{ }_{z} V^{+} \leqslant \lim _{L \rightarrow \infty}{ }_{y} V^{-}(L)+\sigma(\epsilon)
$$

which is (2.30). This completes the proof of (ii).

ThEOREM 2.4. Let (A), (B) hold. Then
(i) $\lim _{M \rightarrow \infty} \lim _{L \rightarrow \infty} V^{+}(M, L)={ }_{y} V^{-}$,
(iii) $\lim _{L \rightarrow \infty} \lim _{M \rightarrow \infty} V^{+}(M, L)={ }_{2} V^{+}$,
(ii) $\lim _{M \rightarrow \infty} \lim _{L \rightarrow \infty} V^{-}(M, L)={ }_{y} V^{-}$,
(iv) ' $\lim _{L \rightarrow \infty} \lim _{M \rightarrow \infty} V^{-}(M, L)={ }_{2} V^{+}$.

Proof. We only prove (i); the others are similar.

By Theorem 2.2(v)

$$
\lim _{L \rightarrow \infty} y_{0} V^{+}(M, L)={ }_{z}^{0} V^{+}(M)
$$

so

$$
\lim _{M \rightarrow \infty} \lim _{L \rightarrow \infty} y_{0} V^{+}(M, L)=\lim _{M \rightarrow \infty}{ }^{2} V^{+}(M) .
$$

By Theorem 2.3(i) 


$$
\lim _{M \rightarrow \infty}{ }_{2} V^{+}(M)={ }_{y} V^{\bar{z}}
$$

Therefore

$$
\lim _{M \rightarrow \infty} \lim _{L \rightarrow \infty} y_{0} V^{+}(M, L)={ }_{y} V^{-}
$$

But, by Lemma 2.6(v)

$$
\lim _{M \rightarrow \infty} \lim _{L \rightarrow \infty} y_{0} V^{+}(M, L)=\lim _{M \rightarrow \infty} \lim _{L \rightarrow \infty} V^{+}(M, L) .
$$

Thus

$$
\lim _{M \rightarrow \infty} \lim _{L \rightarrow \infty} V^{+}(M, L)={ }_{y} V^{-}
$$

Corollary 2.4 .

(i)

$$
\lim _{M \rightarrow \infty} \lim _{L \rightarrow \infty} V^{+}(M, L)=\lim _{M \rightarrow \infty} V^{+}(M),
$$

$$
\begin{aligned}
& \lim _{M \rightarrow \infty} \lim _{L \rightarrow \infty} V^{-}(M, L)=\lim _{M \rightarrow \infty} V^{-}(M), \\
& \lim _{L \rightarrow \infty} \lim _{M \rightarrow \infty} V^{+}(M, L)=\lim _{L \rightarrow \infty} V^{+}(L),
\end{aligned}
$$

$$
\lim _{L \rightarrow \infty} \lim _{M \rightarrow \infty} V^{-}(M, L)=\lim _{L \rightarrow \infty} V^{-}(L) .
$$

We now are in a position to prove Theorem 2.1 which we restate here for convenience.

THEOREM 2.1. Let (A), (B) hold. If

$$
\lim _{M \rightarrow \infty} \lim _{L \rightarrow \infty} V^{+}(M, L)=\lim _{L \rightarrow \infty} \lim _{M \rightarrow \infty} V^{+}(M, L)
$$

then the differential game associated with (1.1)-(1.4) and payoff (1.7) has value $V$; i.e. $V=V^{+}=V^{-}$and

$$
V=\lim _{M \rightarrow \infty} \lim _{L \rightarrow \infty} V^{+}(M, L)=\lim _{L \rightarrow \infty} \lim _{M \rightarrow \infty} V^{+}(M, L)
$$

Proof. We always have

$$
{ }_{z} V^{+} \geqslant V^{+} \geqslant V^{-} \geqslant{ }_{y} V^{-} \text {. }
$$

By Theorem 2.4 and the assumption (2.38), from (2.39) we get

$$
\lim _{L \rightarrow \infty} \lim _{M \rightarrow \infty} V^{+}(M, L)={ }_{z} V^{+} \geqslant V^{+} \geqslant V^{-\geqslant}{ }_{y} V^{-}=\lim _{M \rightarrow \infty} \lim _{L \rightarrow \infty} V^{+}(M, L) .
$$

That is, if (2.38) holds 
$\lim _{L \rightarrow \infty} \lim _{M \rightarrow \infty} V^{+}(M, L)={ }_{z} V^{+}=V^{+}=V^{-}={ }_{y} V^{-}=\lim _{M \rightarrow \infty} \lim _{L \rightarrow \infty} V^{+}(M, L)$.

REMARK 2.3. Again we emphasize that the iterated limits in (2.38) always exist. Note, e.g. that (2.38) holds when either $\lim _{(M, L) \rightarrow(\infty, \infty)} V^{+}(M, L)$ exists or when one of the limits in (2.38) is uniform, e.g. if $\lim _{M \rightarrow \infty} V^{+}(M, L)$ is uniform in $L$.

By Theorem 2.4, $V^{+}(M, L)$ may be replaced by $V^{-}(M, L)$ in (2.38). In fact, if the iterated limits in (2.38) are equal, Theorem 2.4 implies $\lim _{L \rightarrow \infty} \lim _{M \rightarrow \infty} V^{-}(M, L)=\lim _{M \rightarrow \infty} \lim _{L \rightarrow \infty} V^{-}(M, L)$.

REMARK 2.4. Let ${ }_{z, y} V^{+},{ }_{z, y} V^{-}$denote the upper and lower values, respectively, of the differential game associated with (1.1)-(1.4) and payoff (1.7) when both $y$ and $z$ are restricted at each step to choosing control functions which are constants. ${ }_{z, y} V^{+},{ }_{z y} V^{-}$are Fleming's $W^{+}$and $W^{-}$(see [6]).

$$
{ }_{z} V^{+} \geqslant_{z, y} V^{+} \geqslant_{z, y} V^{-} \geqslant_{y} V^{-}
$$

and, if the hypotheses of Theorem 2.1 are valid $V^{+}={ }_{z} V^{+}={ }_{y} V^{-}=V^{-}$, we have

$$
V^{+}={ }_{z} V^{+}={ }_{z, y} V^{+}={ }_{z, y} V^{-}={ }_{y} V^{-}=V^{-}
$$

REMARK 2.5. All of the theorems and lemmas of this section remain valid for more general payoffs of fixed duration of the form

$$
P(y, z)=g(u)+\int_{0}^{T} \int_{0}^{B} h(t, x, u, y, z) d x d t
$$

with suitable continuity restrictions on $g$ and $h$. Furthermore, the results are valid for differential games governed by general parabolic or hyperbolic problems in general domains.

We specialize now to the case when we have differential games govemed by ordinary differential equations and establish a general result holding in this case.

Consider a system of $m$ ordinary differential equations

$$
d x / d t=r(t, x, y, z), \quad t_{0}<t \leqslant T_{0},
$$

with initial condition

$$
x\left(t_{0}\right)=x_{0}
$$

Let $Y, Z$ be compact subsets of some Euclidean spaces $R^{p}, R^{q}$, respectively. Note that $x$ here is not a spatial variable, but represents an $m$-vector solution of (2.41), (2.42).

We assume 
$r(t, x, y, z)$ is continuous in $(t, x, y, z)$

$\in\left[t_{0}, T_{0}\right] \times R^{m} \times Y \times Z ; x \cdot r(t, x, y, z) \leqslant k(t)\left(1+|x|^{2}\right)$

where $\int_{t_{0}}^{T_{0}} k(t) d t<\infty$ and, for any $R>0$

$\left(A_{1}\right)$

$$
|r(t, x, y, z)-r(t, \bar{x}, \bar{y}, \bar{z})|
$$

$$
\leqslant k_{R}(t)(|x-\bar{x}|+|y-\bar{y}|+|z-\bar{z}|)
$$

for all $t \in\left[t_{0}, T_{0}\right], y \in Y, z \in Z,|x|<R,|\bar{x}|<R$

where $k_{R}(t)$ is a function depending on $R$ and satisfying $\int_{t_{0}}^{T_{0}} k_{R}(T) d t<\infty$.

Consider a payoff

$$
P(y, z)=g\left(x\left(T_{0}\right)\right)+\int_{t_{0}}^{T_{0}} h(t, x(t), y(t), z(t)) d t .
$$

Assume

$g(x)$ is continuous in $R^{m}$ and $h(t, x, y, z)$ is Lipschitz continuous in $(t, x, y, z) \in\left[t_{0}, T_{0}\right] \times R^{m} \times Y \times Z$.

Consider the ordinary differential game of fixed duration associated with (2.41), (2.42) and (2.43). All terminology and notations concerning these games will conform to Friedman [1], [2].

When the controls $y, z$ must choose Lipschitz $M, L$ functions on $\left[t_{0}, T_{0}\right]$ we define as above $V^{ \pm}(L), V^{ \pm}(M), V^{ \pm}(M, L)$. We also define similarly ${ }_{z} V^{\delta}$, ${ }_{y} V^{\delta}, \ldots$ etc.

THEOREM 2.5. Consider the differential game of fixed duration associated with (2.41), (2.42) and payoff (2.43). Assume $\left(\mathrm{A}_{1}\right),\left(\mathrm{B}_{1}\right)$ hold and assume the game has value $V, V=V^{+}=V^{-}$. Then, given $\epsilon>0$, there exist $M_{0}, L_{0}$ depending only on $\epsilon, r, g, h$ such that

$$
M \geqslant M_{0}, L \geqslant L_{0} \quad \text { imply }\left|V^{+}(M, L)-V^{+}\right|<\epsilon, \quad\left|V^{-}(M, L)-V^{-}\right|<\epsilon .
$$

Proof. By a modified result of Friedman-Fleming-Elliott-Kalton (see for example [2], [3], [5], [6]) given $\epsilon>0$, there is $\delta_{0}(\epsilon)$ such that

$$
I_{z} V^{\delta}-V^{+}\left|<\epsilon, \quad I_{y} V_{\delta}-V^{-}\right|<\epsilon \text { when } \delta \leqslant \delta_{0}(\epsilon) .
$$

Fix $\delta \leqslant \delta_{0}(\epsilon)$. By Lemma 2.3(i), there exists $L_{0} \equiv L_{1}(\delta, \epsilon)$ such that

$$
z, \delta V^{\eta}+\epsilon \geqslant V^{\eta}(L) \text { for } L \geqslant L_{0}, \eta>0 .
$$

Note that we have placed, without loss of generality, $\epsilon=\epsilon \delta^{2}$.

Let $\eta \rightarrow 0$. Using Lemma 2.2(i) we obtain 


$$
{ }_{2} V^{\delta}+\epsilon \geqslant V^{+}(L) \quad\left(L \geqslant L_{0}\right) .
$$

From (2.44) and (2.45)

$$
V^{+}+2 \epsilon \geqslant V^{+}(L) \quad\left(L \geqslant L_{0}\right) .
$$

Since we always have for each $M \geqslant 0, V^{+}(L) \geqslant V^{+}(M, L) \geqslant V^{-}(M, L) \geqslant$ $V^{-}(M)$ by $(2.46)$ we get

$$
V^{+}+2 \epsilon \geqslant V^{+}(L) \geqslant V^{+}(M, L) \geqslant V^{-}(M, L) \geqslant V^{-}(M) .
$$

From Lemma 2.3(iv) we have the existence of $M_{0} \equiv M_{2}(\delta, \epsilon)$ such that

$$
V_{\eta}(M){ }_{y, \delta} V_{\eta}-\epsilon \text { for } M \geqslant M_{0}, \eta>0 .
$$

Let $\eta \rightarrow 0$ and use Lemma 2.2(iv) in (2.48)

$$
V^{-}(M) \geqslant{ }_{y} V_{\delta}-\epsilon \text { for } M \geqslant M_{0} .
$$

Combining (2.47), (2.49) and using (2.44) we get

$$
\begin{aligned}
& V^{+}+2 \epsilon \geqslant V^{+}(L) \geqslant V^{+}(M, L) \\
& \geqslant V^{-}(M, L) \geqslant V^{-}(M) \geqslant V^{-}-2 \epsilon \quad\left(M \geqslant M_{0}, L \geqslant L_{0}\right) .
\end{aligned}
$$

And since $V^{+}=V^{-}$we have the result. Note we have in fact shown

$$
\begin{aligned}
\lim _{L \rightarrow \infty} V^{+}(L) & =V^{+}=\lim _{(M, L) \rightarrow(\infty, \infty)} V^{+}(M, L) \\
& =\lim _{(M, L) \rightarrow(\infty, \infty)} V^{-}(M, L)=V^{-}=\lim _{M \rightarrow \infty} V^{-}(M) .
\end{aligned}
$$

Remark 2.6. Theorem 2.5 shows that when we know a priori that the ordinary differential game of fixed duration has value then we can in fact choose our controls to be Lipschitz. Theorem 2.5 combined with Theorem 2.1 shows that

$$
\lim _{M \rightarrow \infty} \lim _{L \rightarrow \infty} V^{+}(M, L)=\lim _{L \rightarrow \infty} \lim _{M \rightarrow \infty} V^{+}(M, L)
$$

is a necessary and sufficient condition for value to exist in the case of ordinary differential games.

REMARK 2.7. We can easily modify the proofs of the results in $\$ 2$ so that we can consider the controls to be infinitely differentiable rather than merely Lipschitz. In fact we would have the condition

$$
\lim _{\epsilon \rightarrow 0} \lim _{\gamma \rightarrow 0} V^{+}(\epsilon, \gamma)=\lim _{\gamma \rightarrow 0} \lim _{\epsilon \rightarrow 0} V^{+}(\epsilon, \gamma)
$$

where $V^{+}(\epsilon, \gamma)$ denotes the upper $\delta-(\epsilon, \gamma)$-value when $y, z$ choose control functions given by 


$$
y_{\epsilon}(t)=\int \rho_{\epsilon}(t-s) y(s) d s, \quad z_{\gamma}(t)=\int \rho_{\gamma}(t-s) z(s) d s .
$$

That is, $y_{\epsilon}, z_{\gamma}$ are, respectively, the $\epsilon, \gamma$ mollifiers of $y, z$, where $y, z$ are measurable control functions. Here $\rho_{\epsilon}$ is a suitable $C^{\infty}$ function. See [9] for the theory of mollifiers. This fact is useful in that the infinitely differentiable functions are actually constructable.

REMARK 2.8. By monotonicity

$$
\lim _{M \rightarrow \infty} \lim _{L \rightarrow \infty} V^{+}(M, L)=\sup _{M} \inf _{L} V^{+}(M, L)
$$

and

$$
\lim _{L \rightarrow \infty} \lim _{M \rightarrow \infty} V^{+}(M, L)=\inf _{L} \sup _{M} V^{+}(M, L) .
$$

Thus, by Theorem 2.1, the condition that value exists may be reformulated as

$$
\inf _{L} \sup _{M} V^{+}(M, L)=\sup _{M} \inf _{L} V^{+}(M, L) .
$$

3. Approximating games. In this section we introduce a class of differential games associated with a system of ordinary differential equations whose solution approximates the solution of (1.1)-(1.4). Under the assumptions (A), (B) in the setting of Lipschitz control functions we will show that the upper and lower values of the approximating games converge to $V^{+}(M, L), V^{-}(M, L)$, respectively. Also, we will apply Friedman's results [2], [3] on ordinary differential games to obtain information about our game governed by (1.1)-(1.4), (1.7).

Partition $[0, B]$ of the $x$-axis by a partition $\Pi_{n}$ consisting of $n$ subintervals of equal length $\theta_{n}=B / n$, where $n$ is a positive integer:

$$
\begin{aligned}
\Pi_{n}: 0=x_{0} \leqslant x_{1}<x_{2}<\cdots<x_{i}<x_{i+1}<\cdots<x_{n-1}<x_{n} & =B, \\
x_{i} & =i \cdot \theta_{n} .
\end{aligned}
$$

Consider now the ordinary differential game $G_{n}$ associated with the ordinary differential system

$$
\frac{d w}{d t}=\frac{1}{\theta_{n}^{2}}[A w(t)+\gamma(t)]+F\left({ }^{n} y,{ }^{n} z\right), \quad 0<t \leqslant T,
$$

initial condition

$$
w(0)=w_{0}
$$

and payoff

$$
P_{n}\left({ }^{n} y,{ }^{n} z\right)=\int_{0}^{T} \sum_{i=0}^{n-1} \theta_{n} h\left(t, x_{i}, u_{i}(t), y_{i}(t), z_{i}(t)\right) d t
$$


corresponding to the partition $\Pi_{n}$ of $[0, B]$.

Here we have

$$
\begin{aligned}
& w(t)=\left[\begin{array}{c}
u_{1}(t) \\
u_{2}(t) \\
\vdots \\
u_{n-1}(t)
\end{array}\right]_{n-1 \times 1}, w_{0}=\left[\begin{array}{c}
\phi\left(x_{1}\right) \\
\phi\left(x_{2}\right) \\
\vdots \\
\phi\left(x_{n-1}\right)
\end{array}\right]_{n-1 \times 1}, \\
& \gamma(t)=\left[\begin{array}{c}
u_{0}(t) \\
0 \\
\vdots \\
0 \\
u_{n}(t)
\end{array}\right]_{n-1 \times 1}=\left[\begin{array}{c}
a(t) \\
0 \\
\vdots \\
0 \\
b(t)
\end{array}\right]_{n-1 \times 1}, \\
& A=\left[\begin{array}{rrrrrrrr}
-2 & 1 & 0 & 0 & \cdots & 0 & 0 & 0 \\
1 & -2 & 1 & 0 & \cdots & 0 & 0 & 0 \\
0 & 1 & -2 & 1 & \cdots & 0 & 0 & 0 \\
\vdots & & & & & & & \\
0 & 0 & 0 & 0 & \cdots & 0 & 1 & -2
\end{array}\right]_{n-1 \times n-1}, n^{n}(t)=\left[\begin{array}{c}
y_{0}(t) \\
y_{1}(t) \\
\vdots \\
y_{n-1}(t)
\end{array}\right]_{n \times 1} \\
& n_{z}(t)=\left[\begin{array}{c}
z_{0}(t) \\
z_{1}(t) \\
\vdots \\
z_{n-1}(t)
\end{array}\right]_{n \times 1} \\
& F\left({ }^{n} y,{ }^{n} z\right)=\left[\begin{array}{c}
f\left(y_{1}, z_{1}\right) \\
f\left(y_{2}, z_{2}\right) \\
\vdots \\
f\left(y_{n-1}, z_{n-1}\right)
\end{array}\right]_{n-1 \times 1}
\end{aligned}
$$

Note that (3.1), (3.2) are equivalent to the system of $(n-1)$ ordinary differential equations

$$
\frac{d u_{i}}{d t}=\frac{1}{\theta_{n}^{2}}\left[u_{i-1}(t)+u_{i+1}(t)-2 u_{i}(t)\right]+f\left(y_{i}(t), z_{i}(t)\right),
$$

$$
1 \leqslant i \leqslant n-1
$$




$$
u_{i}(0)=\phi\left(x_{i}\right), \quad 1 \leqslant i \leqslant n-1, \quad u_{0}(t)=a(t), \quad u_{n}(t)=b(t),
$$

for the $n-1$ function $u_{i}(t)$.

In $R^{n+1}$ we use the norm

$$
\|x\|=\left(\sum_{i=0}^{n} \frac{B}{n}\left|x_{i}\right|^{2}\right)^{1 / 2} .
$$

Note that if we let

$$
\begin{aligned}
& f_{n}\left(t, w,{ }^{n} y,{ }^{n} z\right)=\frac{1}{\theta_{n}^{2}}[A w+\gamma]+F\left({ }^{n} y,{ }^{n} z\right), \\
& h_{n}\left(t, w,{ }^{n} y,{ }^{n} z\right)=\sum_{i=0}^{n-1} \theta_{n} h\left(t, x_{i}, u_{i}, y_{i}, z_{i}\right),
\end{aligned}
$$

then assuming conditions $(\mathrm{A}),(\mathrm{B})$ will imply conditions $\left(\mathrm{A}_{1}\right),\left(\mathrm{B}_{1}\right)$ hold for $f_{n}$ and $h_{n}$, for each $n$.

In the above game, $G_{n}, y$ chooses a measurable function $y=y(t, x)$ with values almost everywhere in $Y$ and we place $y_{k}(t)=y\left(t, x_{k}\right), 0 \leqslant k \leqslant n-1$. Then ${ }^{n} y(t)$ is the vector above. Similarly for ${ }^{n} z(t)$. We shall define the concept of solution to (3.1), (3.2) for each choice of ${ }^{n} y,{ }^{n} z$ to be given by the solution of the integral equation

$$
w(t)=w_{0}+\int_{0}^{t} f_{n}\left(s, w(s),{ }^{n} y(s),{ }^{n} z(s)\right) d s, \quad 0 \leqslant t \leqslant T .
$$

A solution then is uniquely determined for each choice of controls ${ }^{n} y,{ }^{n} z$ under the condition (A).

We have need of the

THEOREM 3.1. Let (A), (B) hold. Let $y$ choose $y=y_{M}(t, x) \in$ $Y^{M}([0, T] \times[0, B]), z$ choose $z=z_{L}(t, x) \in Z^{L}([0, T] \times[0, B])$. Place these functions in (1.1) and solve the system (1.1)-(1.4) obtaining a function $u=u(t, x)$ given by (1.6). Put $y_{M k}(t)=y_{M}\left(t, x_{k}\right), z_{L k}(t)=z_{L}\left(t, x_{k}\right), 0 \leqslant$ $k \leqslant n-1$, in (3.1) and solve the system (3.1), (3.2) obtaining a vector $w(t)=$ $\left(u_{1}(t), \ldots, u_{n-1}(t)\right)$. Then, given any $\epsilon>0$, there is an $N$ depending on the functions $f, \phi, a, b$ and the constants $M, L, \epsilon$, such that for each $i=1,2, \ldots$, $n-1$

$$
\left|u(t, x)-u_{i}(t)\right|<\epsilon, \quad 0<t<T, x_{i-1}<x<x_{i},
$$

whenever $n \geqslant N$.

The proof of Theorem 3.1 is given in [7] for the homogeneous case. Our condition (A) on $f, \phi, a, b$ and the Lipschitz continuity of $y_{M}, z_{L}$ insure that 
the proof can be easily carried out in our inhomogeneous case as pointed out in [7].

Definition. A function $g$ defined on $I \times[0, B]$, where $I$ is a subinterval of $[0, T]$ is $x$-piecewise constant corresponding to the partition $\Pi_{n}$ of $[0, B]$ if $g=g(t, x)$ is a constant in the $x$ direction on each subinterval of $\Pi_{n}$; i.e. $g(t, x)$ $=c_{i}(t), x_{i-1}<x \leqslant x_{i}, t \in I, 1 \leqslant i \leqslant n$. If the partition $\Pi_{n}$ is understood we simply say that $g$ is $x$-piecewise constant.

A function $g$ is $x$-piecewise constant with A-slope restriction corresponding to the partition $\Pi_{n}$ of $[0, B]$ if $g=g(t, x)$ is $x$-piecewise constant corresponding to the partition $\Pi_{n}$ and

$$
\left|g\left(t, x_{i-1}\right)-g\left(t, x_{i}\right)\right| \leqslant A\left|x_{i-1}-x_{i}\right|
$$

for all $t \in I, 1 \leqslant i \leqslant n$, where $A$ is a positive constant. If $\Pi_{n}$ is understood we simply say that $g$ is $x$-piecewise constant with $A$-slope restriction.

Denote by $Y^{M}\left(I ; \Pi_{n}\right)$ the class of all functions defined on $I \times[0, B]$ which are Lipschitz (constant $M$ ) in $t$ and which are $x$-piecewise constant with $M$-slope restriction corresponding to the partition $\Pi_{n}$ and which take values in the given compact set $Y$. Similarly, $Z^{L}\left(I ; \Pi_{n}\right)$ is the class of all functions defined in $I \times$ $[0, B]$ which are Lipschitz (constant $L$ ) in $t$, which are $x$-piecewise constant with $L$-slope restriction corresponding to the partition $\Pi_{n}$ and which take values in the given compact set $Z$.

For a given partition $\Pi_{n}$, the terminology and concepts of the ordinary differential game associated with (3.1), (3.2) and payoff (3.3) will conform to Friedman [1], [2]. As such we let ${ }^{n} V^{\delta},{ }^{n} V_{\delta}$ denote the upper and lower $\delta$ values, respectively, for $\delta=T / \alpha, \alpha$ a positive integer.

The defining upper and lower $\delta$-strategies will be denoted by ${ }^{n} \Gamma^{\delta},{ }^{n} \Gamma_{\delta}$, ${ }^{n} \Delta^{\delta},{ }^{n} \Delta_{\delta}$. Then, as in Friedman [1]

$$
\begin{aligned}
& { }^{n} V^{\delta}=\inf _{n_{\Delta_{\delta}}{ }^{n} \Gamma^{\delta}} P_{n}\left[{ }^{n} \Delta_{\delta},{ }^{n} \Gamma^{\delta}\right],{ }^{n} V^{+}=\lim _{\delta \rightarrow 0}{ }^{n} V^{\delta}, \\
& { }^{n} V_{\delta}=\sup _{n_{\Gamma_{\delta}} \inf _{\Delta^{\delta}} P_{n}\left[{ }^{n} \Gamma_{\delta},{ }^{n} \Delta^{\delta}\right],}{ }^{n} V^{-}=\lim _{\delta \rightarrow 0}{ }^{n} V_{\delta} .
\end{aligned}
$$

Note that when $y, z$ are restricted to choosing Lipschitz control functions then the solution $u(t, x)$ of $(1.1)-(1.4)$ given by $(1.6)$ is continuous in $(t, x)$ with $u_{t}(t, x)$ and $u_{x x}(t, x)$ also continuous and $u(t, x)$ then satisfies (1.5) everywhere. Then, by Theorem 3.1 it is easily seen that when $n$ is sufficiently large

$$
\left|\frac{d u_{i}(t)}{d t}-\frac{\partial u(t, x)}{\partial t}\right|<\epsilon, \quad t \in[0, T], x_{i-1}<x \leqslant x_{i},
$$

where $w(t)=\left(u_{1}(t), \ldots, u_{n-1}(t)\right)$ is a solution of (3.1), (3.2). Thus 


$$
\left\|f_{n}\left(t, w,{ }^{n} y,{ }^{n} z\right)\right\| \leqslant\left\|\frac{d w}{d t}\right\|=\sup _{t \in[0, T]}\left(\sum_{i=0}^{n} \frac{B}{n}\left|\frac{d u_{i}(t)}{d t}\right|^{2}\right)^{1 / 2}
$$

But

$$
\lim _{n \rightarrow \infty}\left(\sum_{i=0}^{n} \frac{B}{n}\left|\frac{d u_{i}}{d t}\right|^{2}\right)^{1 / 2}=\int_{0}^{B}\left|\frac{\partial u(t, x)}{\partial t}\right|^{2} d x \leqslant|c(t)|
$$

where $c(t)$ is a continuous function on $[0, T]$, independent of $n$. Hence

$$
\left\|f_{n}\left(t, w,{ }^{n} y,{ }^{n} z\right)\right\| \leqslant \sup _{t \in[0, T]}|c(t)| \leqslant C=\text { constant }
$$

for all controls ${ }^{n} y,{ }^{n} z$ determined from Lipschitz functions. Similarly

$$
\left\|h_{n}\left(t, w,{ }^{n} y,{ }^{n} z\right)\right\| \leqslant C \text {. }
$$

DEFinition. When $y$ is restricted to choosing functions in $Y^{M}\left([0, T] ; \Pi_{n}\right)$ and $z$ is restricted to choosing functions in $Z^{L}\left([0, T] ; \Pi_{n}\right)$, denote by ${ }^{n} V^{\delta}(M, L)$, ${ }^{n} V_{\delta}(M, L)$ the upper and lower, respectively, $\delta-(M, L)$-values of the differential game associated with (3.1), (3.2) (or (3.4), (3.5)) and payoff (3.3).

Thus, if we denote functions in $Y^{M}\left([0, T] ; \Pi_{n}\right)$ by ${ }^{n} y_{M}(t)$, then ${ }^{n} y_{M}(t)$ is the vector

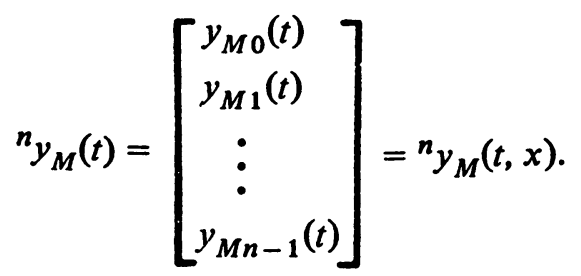

Similarly, if functions in $Z^{L}\left([0, T] ; \Pi_{n}\right)$ are denoted by ${ }^{n} z_{L}(t)$, then ${ }^{n} z_{L}(t)$ is the vector

$$
n_{z_{L}}(t)=\left[\begin{array}{c}
z_{L 0}(t) \\
z_{L 1}(t) \\
\vdots \\
z_{L n-1}(t)
\end{array}\right]={ }^{n} z_{L}(t, x)
$$

Denote the corresponding $\delta$-strategies by ${ }^{n M} \Gamma^{\delta},{ }^{n M} \Gamma_{\delta},{ }^{n L} \Delta^{\delta}$ and ${ }^{n L} \Delta_{\delta}$. As in $\$ 2$ we have

$$
\begin{aligned}
& { }^{n} V^{\delta}(M, L)=\inf _{n \Delta_{\Delta_{\delta}}} \sup _{n M_{\Gamma} \delta} P_{n}\left[{ }^{n L} \Delta_{\delta},{ }^{n M} \Gamma^{\delta}\right], \\
& { }^{n} V_{\delta}(M, L)=\sup _{n M_{\Gamma_{\delta}}} \inf _{n L_{\Delta^{\delta}}} P_{n}\left[{ }^{n M_{\Gamma_{\delta}}},{ }^{n L} \Delta^{\delta}\right],
\end{aligned}
$$


and by (3.10), (3.11), $\lim _{\delta \rightarrow 0}{ }^{n} V^{\delta}(M, L) \equiv{ }^{n} V^{+}(M, L)$ and $\lim _{\delta \rightarrow 0}{ }^{n} V_{\delta}(M, L)$ $\equiv{ }^{n} V^{-}(M, L)$ exist uniformly in $n, M, L$ when (A), (B) hold.

We will use results similar to those stated in $\S 2$ for $V^{\delta}(M, L), V_{\delta}(M, L)$. The proofs will be similar. Also we shall use definitions and results for ${ }^{n} V^{\delta}(M)$, ${ }^{n} V_{\delta}(M),{ }^{n} V^{\delta}(L),{ }^{n} V_{\delta}(L)$ and limits as $\delta \rightarrow 0$. The notation used is obvious.

Definition. Let ${ }^{n} \bar{V}^{\delta}(M, L)$ denote the upper $\delta-(M, L)$-value of the differential game associated with (1.1)-(1.4) and payoff (1.7) when the players $y, z$ are restricted to choosing control functions which are members of $Y^{M}\left([0, T] ; \Pi_{n}\right)$ respectively. Thus

$$
{ }^{n} \bar{V}^{\delta}(M, L)=\inf _{n L_{\Delta_{\delta}}} \sup _{n M_{\Gamma^{\delta}}} P\left[{ }^{n L} \Delta_{\delta},{ }^{n M^{\delta}} \Gamma^{\delta}\right] .
$$

Similarly we define

$$
{ }^{n} \bar{V}_{\delta}(M, L)=\sup _{n M_{\Gamma_{\delta}}} \lim _{n L_{\Delta} \delta} P\left[{ }^{n M} \Gamma_{\delta},{ }^{n L} \Delta^{\delta}\right]
$$

Again we use results similar to $\S 2$. For example when (A), (B) hold

$$
\begin{aligned}
& { }^{n} \bar{V}^{+}(M, L) \equiv \lim _{\delta \rightarrow 0}{ }^{n} \bar{V}^{\delta}(M, L), \\
& { }^{n} \bar{V}^{-}(M, L) \equiv \lim _{\delta \rightarrow 0}{ }^{n} \bar{V}_{\delta}(M, L) .
\end{aligned}
$$

We now prove various lemmas concerning the intermediate games.

Lemma 3.1. Let (A), (B) hold. Given $\epsilon>0$, there is an $N$ depending on $\epsilon, f, \phi, a, b, h, M, L$, such that when $n \geqslant N$

$$
\left.\right|^{n} \bar{V}^{+}(M, L)-V^{+}(M, L) \mid<\epsilon,
$$

and

$$
\left.\right|^{n} \bar{V}-(M, L)-V^{-}(M, L) \mid<\epsilon .
$$

Proof. We only show (3.14); (3.15) is similar.

Since

$$
V^{\delta}(M, L)=\inf _{L_{\Delta_{\delta}}} \sup _{M_{\Gamma^{\delta}}} P\left[{ }^{L} \Delta_{\delta},{ }^{M} \Gamma^{\delta}\right],
$$

for $\epsilon>0$ given, there is an upper $\delta-(M)$-strategy ${ }^{M} \tilde{\Gamma}^{\delta}$ for $y$ such that

$$
V^{\delta}(M, L) \leqslant P\left[z_{L},{ }^{M} \tilde{\Gamma}^{\delta}\left(z_{L}\right)\right]+\epsilon
$$

for all controls $z_{L} \in Z^{L}([0, T] \times[0, B])$.

Given any control $z_{L}=z_{L}(t, x) \in Z^{L}([0, T] \times[0, B])$, let $\left(z_{L}, \tilde{y}_{M}^{\delta}\right)$ 
denote the outcome of $\left(z_{L},{ }^{M} \widetilde{\Gamma}^{\delta}\left(z_{L}\right)\right)$. Note that $\widetilde{y}_{M}^{\delta}=\tilde{y}_{M}^{\delta}(t, x) \in$ $Y^{M}([0, T] \times[0, B])$.

From well-known properties of equicontinuous classes of functions we can define a $\delta$-strategy ${ }^{n}{ }^{M} \widetilde{\Gamma}^{\delta}$ for $y$ having the following property:

Given any control $z_{L}=z_{L}(t, x)$, there is a constant $N^{1}=N^{1}(\epsilon, M, L)$ such that

$$
\left\|\tilde{y}_{M}^{\delta}-{ }^{n} \tilde{y}_{M}^{\delta}\right\|_{L}^{1}<\epsilon \text { when } n \geqslant N^{1}
$$

where ${ }^{n} \widetilde{y}_{M}^{\delta}$ is the $y$-outcome of $\left(z_{L},{ }^{n M} \widetilde{\Gamma}^{\delta}\left(z_{L}\right)\right.$ and ${ }^{n} \widetilde{y}_{M}^{\delta} \in Y^{M}\left([0, T] ; \Pi_{n}\right)$.

Let $\widetilde{u}_{M}^{\delta}$ denote the solution of (1.1)-(1.4) corresponding to $\left(\tilde{y}_{M}^{\delta}, z_{L}\right)$ and let ${ }^{n} \tilde{u}_{M}^{\delta}$ denote the solution of (1.1)-(1.4) corresponding to $\left({ }^{n} \tilde{y}_{M}^{\delta}, z_{L}\right)$. We have by (1.6) and the uniform continuity of $f$ and

$$
\begin{aligned}
\mid \tilde{u}_{M}^{\delta}(t, x)- & n \tilde{u}_{M}^{\delta}(t, x) \mid \\
& \leqslant \int_{0}^{T} \int_{0}^{B} G(t, x ; \tau, \xi) \mid f\left(y_{M}(\tau, \xi), z_{L}(\tau, \xi)\right) \\
& -f\left({ }^{n} y_{M}(\tau, \xi) z_{L}(\tau, \xi)\right) \mid d \xi d \tau
\end{aligned}
$$

the existence of a positive function $\sigma(\zeta) \rightarrow 0$ as $\zeta \rightarrow 0$ such that

$$
\left\|\tilde{u}_{M}^{\delta}(t, x)-{ }^{n} \tilde{u}_{M}^{\delta}(t, x)\right\|_{\infty} \leqslant \sigma(n)<\epsilon \text { when } n \geqslant N^{1}
$$

Thus, since $h$ is continuous, there is another positive function $\sigma(\zeta) \rightarrow 0$ as $\zeta \rightarrow \infty$, independent of $\delta$ or any controls such that by (3.17), (3.18)

$$
\left|P\left(\tilde{y}_{M}^{\delta}, z_{L}\right)-P\left(n \tilde{y}_{M}^{\delta}, z_{L}\right)\right| \leqslant \sigma(n)<\epsilon
$$

when $n \geqslant N^{1}$ for all controls $z_{L}$.

By (3.16) we have using (3.19) that when $n \geqslant N^{1}$

$$
V^{\delta}(M, L) \leqslant P\left[z_{L},{ }^{n M} \tilde{\Gamma}^{\delta}\left(z_{L}\right)\right]+2 \epsilon
$$

for all controls $z_{L} \in Z^{L}([0, T] \times[0, B])$.

We must show (3.20) holds for all controls ${ }^{n} z_{L} \in Z^{L}\left([0, T] ; \Pi_{n}\right)$. By using $\epsilon$-nets for $Z^{L}([0, T] \times[0, B])$ and recalling the definition of $Z^{L}\left([0, T] ; \Pi_{n}\right)$ it is easily seen that $Z^{L}\left([0, T] ; \Pi_{n}\right)$ is a compact subset of $L^{1}([0, T] \times[0, B])$ independently of $n$.

Given ${ }^{n} z_{L} \in Z^{L}\left[(0, T] ; \Pi_{n}\right)$, let $z_{L}$ denote the function in $Z^{L}([0, T] \times[0, B])$ constructed from ${ }^{n} z_{L}$ by joining the midpoints of each step of ${ }^{n} z_{L}$ by a linear function. We know that $z_{L}$ constructed in such a manner is indeed in $Z^{L}([0, T] \times[0, B])$ because of the $L$-slope restriction on ${ }^{n} z_{L}$. Thus $z_{L}$ will have Lipschitz constant $L$ independent of $n$. Thus, there is a constant 
$N^{11}(\epsilon, M, L)$ such that

$$
\left\|{ }^{n} z_{L}-z_{L}\right\|_{L^{1}} \leqslant C \theta_{n} L<\epsilon \text { when } n \geqslant N^{11}
$$

where $C$ is a constant depending only on the control set $Z$. Note that by compactness of $Z^{L}\left([0, T] ; \Pi_{n}\right)$ we can determine $N^{11}$ as independent of which ${ }^{n} z_{L} \in Z^{L}\left([0, T] ; \Pi_{n}\right)$ we choose.

Define for any control ${ }^{n} z_{L}$ the upper $\delta$-strategy ${ }^{n M} \widetilde{\widetilde{\Gamma}}^{\delta}$ for $y$ as follows:

$$
\begin{aligned}
& \text { If }\left\|^{n} z_{L}-z_{L}\right\|_{L^{1}}<\epsilon\left(n \geqslant N^{11}\right) \text { then } \\
& { }^{n M} \widetilde{\Gamma}^{\delta}\left({ }^{n} z_{L}\right)={ }^{n M} \widetilde{\Gamma}^{\delta}\left(z_{L}\right)={ }^{n} \tilde{y}_{M}^{\delta} .
\end{aligned}
$$

Note that we are able to do this with $N^{11}$ independent of $\delta$ since the approximation is in the $x$-variable, not $t$.

Thus, by (3.21), (3.22) and the continuity of $h$, there is another positive function independent of $\delta$ or controls, $\sigma(\zeta)$, with $\sigma(\zeta) \rightarrow 0$ as $\zeta \rightarrow \infty$ such that, for all controls ${ }^{n} z_{L}$,

$$
\left|P\left({ }^{n} \tilde{y}_{M}^{\delta}, z_{L}\right)-P\left({ }^{n} \tilde{y}_{M}^{\delta},{ }^{n} z_{L}\right)\right| \leqslant \sigma(n)<\epsilon \text { when } n \geqslant N^{11} .
$$

Let $n \geqslant N=N(\epsilon, f, h, \phi, a, b, M, L)=\max \left(N^{1}, N^{11}\right)$. Then, by (3.23), (3.20) we have for all ${ }^{n} z_{L} \in Z^{L}\left([0, T] ; \Pi_{n}\right)$

$$
V^{\delta}(M, L) \leqslant P\left[{ }^{n} z_{L},{ }^{n M} \widetilde{\Gamma}^{\delta}\left({ }^{n} z_{L}\right)\right]+3 \epsilon \quad(n \geqslant N) .
$$

This implies $V^{\delta}(M, L) \leqslant{ }^{n} \bar{V}^{\delta}(M, L)+3 \epsilon(n \geqslant N)$ so that $V^{+}(M, L) \leqslant$ ${ }^{n} \bar{V}^{+}(M, L)+3 \epsilon$. Similarly we can show $V^{+}(M, L) \geqslant{ }^{n} \bar{V}^{+}(M, L)-3 \epsilon$ when $n \geqslant N$ and thus (3.14) is established.

The proof of the following is similar to the proof of Lemma 3 in [8].

Lemm A 3.2. Let (A), (B) hold. Let ${ }^{n} u(t, x)$ denote the solution of (1.1)(1.4) when $y$ chooses

$$
{ }^{n} y_{M}={ }^{n} y_{M}(t)={ }^{n} y_{M}(t, x) \in Y^{M}\left([0, T] ; \Pi_{n}\right)
$$

and $z$ chooses

$$
{ }^{n} z_{L}={ }^{n} z_{L}(t)={ }^{n} z_{L}(t, x) \in Z^{L}\left([0, T] ; \Pi_{n}\right) .
$$

Let $w(t)$ denote the solution of (3.1), (3.2) corresponding to the controls $y_{M k}(t)={ }^{n} y_{M}\left(t, x_{k}\right), z_{L k}(t)={ }^{n} z_{L}\left(t, x_{k}\right) \quad(1 \leqslant k \leqslant n-1)$. We also write these as $u\left({ }^{n} y_{M},{ }^{n} z_{L}\right), w\left({ }^{n} y_{M},{ }^{n} z_{L}\right)$, respectively. Then, given $\epsilon>0$, there is a constant $N=N(\epsilon, h, f, \phi, a, b, M, L)$ such that

$$
\left|P\left(u\left({ }^{n} y_{M},{ }^{n} z_{L}\right)\right)-P_{n}\left(w\left({ }^{n} y_{M},{ }^{n} z_{L}\right)\right)\right|<\epsilon
$$

whenever $n \geqslant N$. 
Proof. By (1.7) and (3.3)

$$
\begin{aligned}
& \left|P\left(u\left({ }^{n} y_{M},{ }^{n} z_{L}\right)\right)-P_{n}\left(w\left({ }^{n} y_{M},{ }^{n} z_{L}\right)\right)\right| \\
& =\mid \int_{0}^{B} \int_{0}^{T} h\left(t, x, u(t, x),{ }^{n} y_{M}(t, x),{ }^{n} z_{L}(t, x)\right) d x d t \\
& \quad-\int_{0}^{T} \sum_{i=0}^{n-1} \theta_{n} h\left(t, x_{i}, u_{i}(t), y_{M i}(t), z_{L i}(t)\right) d t \mid \\
& \leqslant \int_{0}^{T} \mid \sum_{i=0}^{n-1} \int_{x_{i}}^{x_{i+1}} h\left(t, x, u(t, x), y_{M i}(t), z_{L i}(t)\right) d x \\
& \quad-\sum_{i=0}^{n-1} \theta_{n} h\left(t, x_{i}, u_{i}(t), y_{M i}(t), z_{L i}(t)\right) \mid d t \\
& \leqslant \sum_{i=0}^{n-1} \int_{0}^{T} \int_{x_{i}}^{x_{i+1}} \mid h\left(t, x, u(t, x), y_{M i}(t), z_{L i}(t)\right) \\
& \quad-h\left(t, x_{i}, u_{i}(t), y_{M i}(t), z_{L i}(t)\right) \mid d x d t \\
& \leqslant \sum_{i=0}^{n-1} \int_{0}^{T} \int_{x_{i}}^{x_{i+1}} \mid h\left(t, x, u(t, x), y_{M i}(t), z_{L i}(t)\right) \\
& \quad-h\left(t, x, u_{i}(t), y_{M i}(t), z_{L i}(t)\right) \mid d x d t \\
& \quad+\sum_{i=0}^{n-1} \int_{0}^{T} \int_{x_{i}}^{x_{i+1}} \mid h\left(t, x, u_{i}(t), y_{M i}(t), z_{L i}(t)\right) \\
& \quad-h\left(t, x_{i}, u_{i}(t), y_{M i}(t), z_{L i}(t)\right) \mid d x d t .
\end{aligned}
$$

Using now the continuity of $h$ and Theorem 3.1 the lemma follows.

Corollary 3.1. Let (A), (B) hold. Then, given $\epsilon>0$, there is a constant $N=N(\epsilon, f, h, \phi, a, b, h, M, L)$ such that

$$
\begin{array}{ll}
\left|{ }^{n} \bar{V}^{+}(M, L)-{ }^{n} V^{+}(M, L)\right|<\epsilon & (n \geqslant N), \\
\left|{ }^{n} \bar{V}^{-}(M, L)-{ }^{n} V^{-}(M, L)\right|<\epsilon & (n \geqslant N) .
\end{array}
$$

THEOREM 3.2. Let (A), (B) hold. Given $\epsilon>0$, there is a constant $N=$ $N(\epsilon, f, \phi, a, b, h, M, L)$ such that

$$
\begin{array}{ll}
\left|V^{+}(M, L)-{ }^{n} V^{+}(M, L)\right|<\epsilon & (n \geqslant N), \\
\left|V^{-}(M, L)-{ }^{n} V^{-}(M, L)\right|<\epsilon & (n \geqslant N) .
\end{array}
$$

Theorem 3.2 follows immediately from Lemma 3.2 and Corollary 3.1. 
THEOREM 3.3. Let (A), (B) hold. Consider the ordinary differential game associated with (3.1), (3.2) (or (3.4), (3.5)) and payoff (3.3). Assume the game has value for every $n:{ }^{n} V^{+}={ }^{n} V^{-}={ }^{n} V$. Then

$$
\lim _{L \rightarrow \infty} \lim _{M \rightarrow \infty}{ }^{n} V^{+}(M, L)=\lim _{M \rightarrow \infty} \lim _{L \rightarrow \infty}{ }^{n} V^{+}(M, L)={ }^{n} V={ }^{n} V^{+}={ }^{n} V^{-}
$$

uniformly in $n$.

Proof. By (3.10), (3.11)

$$
\lim _{L \rightarrow \infty} \lim _{M \rightarrow \infty}{ }^{n} V^{+}(M, L), \quad \lim _{M \rightarrow \infty} \lim _{L \rightarrow \infty}{ }^{n} V^{+}(M, L)
$$

exist uniformly in $n$.

By Theorem 2.4 and the Friedman-Elliott-Kalton-Fleming theorem [2], [3], [5], [6]

$$
\begin{aligned}
{ }_{z}^{n} V^{+} & ={ }^{n} V^{+}=\lim _{L \rightarrow \infty} \lim _{M \rightarrow \infty}{ }^{n} V^{+}(M, L) \\
& \geqslant{ }_{y}^{n} V^{-}={ }^{n} V^{-}=\lim _{M \rightarrow \infty} \lim _{L \rightarrow \infty}{ }^{n} V^{+}(M, L) .
\end{aligned}
$$

By hypothesis ${ }^{n} V^{+}={ }^{n} V^{-}$and the result follows. We only must explain how we mean ${ }_{z}^{n} V^{+},{ }_{y}^{n} V^{-}$. Note that in the proof of Theorem $2.4, M, L$ depend only on $\delta, \epsilon$ and $\delta$ is independent of $n$. Thus (3.27) holds uniformly in $n$.

We define the following with $B=T$ although the results hold if $B \neq T$.

Given positive integers $\alpha, \beta, n$, let $\delta=T / \alpha, \eta=T / \beta$ and $\Pi_{n}$ be the partition of $[0, B]$ given by $\Pi_{n}: 0=x_{0} \leqslant x_{1}<\cdots<x_{n-1}<x_{n}=B, x_{i}=$ $\theta_{n} \cdot i, \theta_{n}=B / n$.

Then we define similarly as in $\S 2$,

(i) ${ }_{z}^{n} V^{\delta}$-upper $\delta$-value of the game associated with (3.1), (3.2) and payoff (3.3) when, at each $\delta$-subinterval, $z$ chooses a measurable function $z=z(t, x)$ constant on $\delta$-squares and then we place ${ }^{n} z(t)=\left(z_{0}(t), \ldots, z_{n-1}(t)\right)$ where $z_{k}(t)=z\left(t, x_{k}\right) \quad(0 \leqslant k \leqslant n-1)$. The player $y$ chooses arbitrary measurable vectors determined from measurable functions $y=y(t, x)$.

$$
{ }_{z}^{n} V^{\delta}=\inf _{n z} \sup _{n_{\delta}} P_{n}\left[{ }^{n z} \Delta_{\delta},{ }^{n} \Gamma^{\delta}\right] .
$$

Thus, in ${ }_{z, \delta}^{n} V^{\eta}$, by the restriction placed on $z, z$ remains independent of $n$.

(ii) ${ }_{z, \delta}^{n} V^{\eta}$-upper $\eta$-value of the game associated with (3.1), (3.2) and payoff (3.3) when, at each $\eta$-subinterval, $z$ chooses a measurable function $z=$ $z(t, x)$ constant on $\delta$-squares and then we place ${ }^{n} z(t)=\left(z_{0}(t), \ldots, z_{n-1}(t)\right)$ where $z_{k}(t)=z\left(t, x_{k}\right) \quad(0 \leqslant k \leqslant n-1)$. The player $y$ chooses measurable vectors determined from measurable functions $y=y(t, x)$.

$$
z,{ }_{z}^{n} V^{\eta}=\inf _{n z, \delta} \sup _{\Delta_{\eta}} P_{\Gamma^{\eta}} P_{n}\left[{ }^{n z, \delta} \Delta_{\eta},{ }^{n} \Gamma^{\eta}\right] .
$$


Thus, in ${ }_{z, \delta}^{n} V^{\eta}$, by the restriction placed on $z, z$ remains independent of $n$.

Similarly we define ${ }_{y}^{n} V^{\delta}, y,{ }_{\delta}^{n} V^{\eta},{ }_{y}^{n} V_{\delta},{ }_{y, \delta}^{n} V_{\eta},{ }_{z, \delta}^{n} V_{\eta},{ }_{z}^{n} V_{\delta}$, etc. With these definitions, all of the lemmas and theorems of $\$ 2$ hold independent of $n$.

4. The Isaacs condition for games with partial differential equations. Consider the differential game associated with (1.1)-(1.4) and payoff (1.7). Let

$$
\begin{array}{r}
H^{+}(t, u, p) \equiv \min _{z \in Z} \max _{y \in Y}\left\{p \cdot f(y, z)+\int_{0}^{B} h(t, x, u, y, z) d x\right\} \\
\left(p \in R^{1}\right),
\end{array}
$$

$$
H^{-}(t, u, p) \equiv \max _{y \in Y} \min _{z \in Z}\left\{p \cdot f(y, z)+\int_{0}^{B} h(t, x, u, y, z) d x\right\} \underset{\left(p \in R^{1}\right) .}{ }
$$

Theorem 4.1. Let (A), (B) hold. Suppose $H^{+}(t, u, p)=H^{-}(t, u, p)$ for each $(t, u, p) \in[0, T] \times R^{1} \times R^{1}$. Then the differential game associated with (1.1)-(1.4) and payoff (1.7) has value $V$, i.e. $V=V^{+}=V^{-}$.

Proof. By Theorem 2.1 it is enough to show that

$$
\lim _{L \rightarrow \infty} \lim _{M \rightarrow \infty} V^{+}(M, L)=\lim _{M \rightarrow \infty} \lim _{L \rightarrow \infty} V^{+}(M, L) .
$$

Define for $p_{n} \in R^{n}$

$$
\begin{aligned}
& H_{n}^{+}\left(t, w, p_{n}\right) \equiv \min _{n_{z}} \max _{n_{y}}\left\{p_{n} \cdot f_{n}\left(t, w,{ }^{n} y, n_{z}\right)+h_{n}\left(t, w,{ }^{n} y,{ }^{n} z\right)\right\} \\
& H_{n}^{-}\left(t, w, p_{n}\right) \equiv \max _{n_{y}} \min _{n_{z}}\left\{p_{n} \cdot f_{n}\left(t, w,{ }^{n} y, n_{z}\right)+h_{n}\left(t, w,{ }^{n} y,{ }^{n} z\right)\right\}
\end{aligned}
$$

where $f_{n}, h_{n}$ are given by (3.6), (3.7), ${ }^{n} y=\left(y_{0}, \ldots, y_{n-1}\right), n_{z}=\left(z_{0}, \ldots\right.$, $\left.z_{n-1}\right)$. By hypothesis $H^{+}(t, u, p)=H^{-}(t, u, p)$. This implies for each $n$

$$
H_{n}^{+}\left(t, w, p_{n}\right)=H_{n}^{-}\left(t, w, p_{n}\right), \quad\left(t, w, p_{n}\right) \in[0, T] \times R^{n} \times R^{n}
$$

That is, the Isaacs' condition holds for the ordinary differential game associated with (3.1), (3.2) and payoff (3.3). Thus, by the Friedman theorem claiming existence of value when (4.7) holds (see, for example, [2], [3]) we have for each $n$

$$
{ }^{n} V={ }^{n} V^{+}={ }^{n} V^{-} \text {. }
$$

Thus, by Theorem 3.3,

$$
\lim _{M \rightarrow \infty} \lim _{L \rightarrow \infty}{ }^{n} V^{+}(M, L)=\lim _{L \rightarrow \infty} \lim _{M \rightarrow \infty}{ }^{n} V^{+}(M, L)={ }^{n} V^{+}
$$

Thus, by Theorem 3.2, and since $\lim _{n \rightarrow \infty}{ }^{n} V^{+}$can easily be seen to exist, we have 


$$
\lim _{M \rightarrow \infty} \lim _{L \rightarrow \infty} V^{+}(M, L)=\lim _{L \rightarrow \infty} \lim _{M \rightarrow \infty} V^{+}(M, L)=\lim _{n \rightarrow \infty}{ }^{n} V^{+}=\lim _{n \rightarrow \infty}{ }^{n} V
$$

and so (4.3) holds.

Thus, we have by Theorems 2.1 and 4.1 if the Isaacs condition holds

$$
V=V^{+}=V^{-}=\lim _{M \rightarrow \infty} \lim _{L \rightarrow \infty} V^{+}(M, L)=\lim _{L \rightarrow \infty} \lim _{M \rightarrow \infty} V^{+}(M, L)=\lim _{n \rightarrow \infty}{ }^{n} V \text {. }
$$

REMARK 4.1. The results of $\S \S 3$ and 4 can be easily extended to differential games of fixed duration with partial differential equations for more general payoffs of the form

$$
P(y, z)=g(u)+\int_{0}^{T} \int_{0}^{B} h(t, x, u, y, z) d x d t
$$

with suitable continuity restrictions on $g, h$. Also the results are valid for games governed by general parabolic or hyperbolic problems in general domains with suitable difference approximations.

REMARK 4.2. The results of $\S \S 3$ and 4 extend easily to the important case when the control functions appear also in the initial and/or boundary conditions.

\section{BIBLIOGRAPHY}

1. A. Friedman, Differential games, Wiley, New York, 1971.

2. - Differential games, National Science Foundation Regional Research Conference on Differential Games and Control Theory, June 4-8, 1973.

3. Upper and lower values of differential games, J. Differential Equations 12 (1972), 462-473; correction, ibid. 13 (1973).

4. - Comparison theorems for differential games. I, J. Differential Equations 12 (1972), 162-172.

5. R. J. Elliott and N. J. Kalton, The existence of value in differential games, Mem. Amer. Math. Soc. No. 126 (1972).

6. W. H. Fleming, The convergence problem for differential games. II, Advances in Game Theory, Ann. of Math. Studies, no. 52, Princeton Univ. Press, Princeton, N.J., 1964, pp. 195-210. MR 29 \#6947.

7. A. N. Tihonov and A. A. Samarskii, The equations of mathematical physics, $2 \mathrm{nd}$ ed., GITTL, Moscow, 1953; English transl., Partial differential equations of mathematical physics. Vol. II, Holden-Day, San Francisco, Calif., 1967. MR 16, 364; 35 \#6957.

8. B. M. Budak, E. M. Berkovič and E. N. Solov'eva, Difference approximations in optimal control problems, SIAM J. Control 7 (1969), 18-31. MR 39 \#4721.

9. S. Agmon, Lectures on elliptic boundary value problems, Van Nostrand Math. Studies, no. 2, Van Nostrand, Princeton, N.J., 1965. MR 31 \#2504.

DEPARTMENT OF MATHEMATICS, GEORGIA INSTITUTE OF TECHNOLOGY, ATLANTA, GEORGIA 30332 